\title{
T-duality diagram for a weakly curved background
}

\author{
Ljubica Davidovića ${ }^{a}$ Bojan Nikolić ${ }^{b}$, Branislav Sazdovićc $^{c}$ \\ Institute of Physics, University of Belgrade, Belgrade, Serbia
}

Received: 18 June 2014 / Accepted: 23 November 2015 / Published online: 7 December 2015

(C) The Author(s) 2015. This article is published with open access at Springerlink.com

\begin{abstract}
In one of our previous papers we generalized the Buscher T-dualization procedure. Here we will investigate the application of this procedure to the theory of a bosonic string moving in the weakly curved background. We obtain the complete T-dualization diagram, connecting the theories which are the result of the T-dualizations over all possible choices of the coordinates. We distinguish three forms of the T-dual theories: the initial theory, the theory obtained Tdualizing some of the coordinates of the initial theory and the theory obtained T-dualizing all of the initial coordinates. While the initial theory is geometric, all the other theories are non-geometric and additionally non-local. We find the T-dual coordinate transformation laws connecting these theories and show that the set of all T-dualizations forms an Abelian group.
\end{abstract}

\section{Introduction}

T-duality is a property of string theory that was not encountered in any point particle theory [1-4]. Its discovery was surprising, because it implies that there exist theories, defined for essentially different geometries of the compactified dimensions, which are physically equivalent. The origin of $\mathrm{T}$ duality is seen in the possibility that, unlike a point particle, the string can wrap around compactified dimensions. But, no matter if one dimension is compactified on a circle of radius $R$ or rather on a circle of radius $l_{s}^{2} / R$, where $l_{s}$ is the fundamental string length scale, the theory will describe the string with the same physical properties. The investigation of T-duality does not cease to provide interesting new physical implications.

Work supported in part by the Serbian Ministry of Education, Science and Technological Development, under contract No. 171031.

\footnotetext{
a e-mail: ljubica@ipb.ac.rs

b e-mail: bnikolic@ipb.ac.rs

c e-mail: sazdovic@ipb.ac.rs
}

The prescription for obtaining the equivalent T-dual theories is given by the Buscher T-dualization procedure $[5,6]$. The procedure is applicable along the isometry directions, which allows the investigation of the backgrounds which do not depend on some coordinates. It is found that T-duality transforms geometric backgrounds to the non-geometric backgrounds with $Q$ flux which are locally well defined, and these to different types of non-geometric backgrounds, backgrounds with $R$ flux which are not well defined even locally $[7,8]$. A similar prescription can be used to obtain fermionic T-duality $[9,10]$. It is argued that the better understanding of T-duality should be sought for by doubling the coordinates, investigating the theories in which the background fields depend on both the usual space-time coordinates and their doubles [11-14], which would make the T-duality a manifest symmetry.

T-duality enables the investigation of the closed string non-commutativity. The coordinates of the closed string are commutative when the string moves in a constant background. In a 3-dimensional space with the Kalb-Ramond field depending on one of the coordinates, successive Tdualizations along isometry directions lead to a theory with Q flux and the non-commutative coordinates [15-17]. The novelty in the research is the generalized T-dualization procedure, realized in [18], addressing the bosonic string moving in the weakly curved background-constant gravitational field and coordinate dependent Kalb-Ramond field with an infinitesimal field strength. The non-commutativity characteristics of a closed string moving in the weakly curved background was considered in [19].

The generalized procedure is applicable to all the spacetime coordinates on which the string backgrounds depend. In Ref. [18], it was first applied to all initial coordinates, which produces a T-dual theory; it was then applied to all the T-dual coordinates and the initial theory was obtained. In this paper, we will investigate the application of the generalized T-dualization procedure to an arbitrary set of coordinates. Let us denote the T-dualization along the direction $x^{\mu}$ by $T^{\mu}$ and 
the T-dualization along dual direction $y_{\mu}$ by $T_{\mu}$. Choosing $d$ arbitrary directions, we denote

$\mathcal{T}^{a}=\circ_{n=1}^{d} T^{\mu_{n}}, \quad \mathcal{T}^{i}=\circ_{n=d+1}^{D} T^{\mu_{n}}, \quad \mathcal{T}=\circ_{n=1}^{D} T^{\mu_{n}}$,

$\mathcal{T}_{a}=\circ_{n=1}^{d} T_{\mu_{n}}, \quad \mathcal{T}_{i}=\circ_{n=d+1}^{D} T_{\mu_{n}}, \quad \tilde{\mathcal{T}}=\circ_{n=1}^{D} T_{\mu_{n}}$,

where $\mu_{n} \in(0,1, \ldots, D-1)$, and $\circ$ denotes the composition of T-dualizations. We will apply T-dualizations (1) to the initial theory, and T-dualizations (2) to its completely Tdual theory (obtained in [18]). We will prove the following composition laws:

$\mathcal{T}^{i} \circ \mathcal{T}^{a}=\mathcal{T}, \quad \mathcal{T}_{i} \circ \mathcal{T}_{a}=\tilde{\mathcal{T}}, \quad \mathcal{T}_{a} \circ \mathcal{T}^{a}=1$,

where 1 denotes the identical transformation (T-dualization not performed). Therefore, the elements $1, \mathcal{T}^{a}$ and $\mathcal{T}_{a}$, with $d=1, \ldots, D$, form an Abelian group. We will find the explicit form of the resulting theories and the corresponding T-dual coordinate transformation laws. These results complete the T-dualization diagram connecting all the theories T-dual to the initial theory.

Throughout the whole article (except for Sect. 9) we assume that the Kalb-Ramond field depends on all coordinates. In that case all T-dual theories, except the initial theory, are non-geometric and non-local because they depend on the variable $V^{\mu}$, which is a line integral of the derivatives of the dual coordinates. To all of these theories there corresponds a flux which is of the same type as the $R$ flux unlike the nongeometric theories with $Q$ flux, which have a local geometric description.

In Sects. 9.1 and 9.2, we present an example of the 3dimensional torus, $T^{3}$ with H-flux, where Kalb-Ramond field depends only on coordinate $x^{3}$. Then T-dualizations along the isometry directions $x^{1}$ and $x^{2}$ lead to geometric background and the T-dualization along $x^{3}$ leads to nongeometric background. In Sect. 9.1 putting $D=3, d=1,2$ with $B_{\mu \nu}$ depending on $x^{3}$ we reproduce the T-duality chain of Refs. [15-17].

In Sect. 9.2 we will compare the results of our paper with those of Ref. [8]. In our manuscript, the background fields' argument, the variable $V^{\mu}$, incorporates all features of the non-geometric spaces. First, as pointed out in Ref. [8] it "eludes a geometric description even locally" because it is a line integral of the derivative. Second, we obtain nonassociativity and breaking of Jacobi identity typical for the so called R-flux backgrounds. In Sect. 9.3 we present example of the 4-dimensional torus $T^{4}$ to generalize the case of Ref. [20] to critical surface.

The generalized T-dualization procedure originates from the Buscher T-dualization procedure. The first rule in the prescription is to replace the derivatives with the covariant derivatives. The new point in the prescription is the replacement of the coordinates in the background fields' argument with the invariant coordinates. The invariant coordinates are defined as the line integrals of the covariant derivatives of the original coordinates. Both covariant derivatives and invariant coordinates are defined using the gauge fields. These fields should be nonphysical, so one requires that their field strength should be zero. This is realized by adding the corresponding Lagrange multipliers' terms. As a consequence of the translational symmetry one can fix the coordinates along which the T-dualization is performed and obtain a gauge fixed action. An important cross-way in the T-dualization procedure is determined by the equations of motion of the gauge fixed action. Two equations of motion obtained varying this action are used to direct the procedure either back to the initial action or forward to the T-dual action. For the equation of motion obtained varying the action over the Lagrange multipliers, the gauge fixed action reduces to the initial action. For the equation of motion obtained varying the action over the gauge fields one obtains the T-dual theory. Comparing the solutions for the gauge fields in these two directions, one obtains the T-dual coordinate transformation laws.

\section{T-duality in the weakly curved background}

Let us consider the closed bosonic string propagating in the background with metric field $G_{\mu \nu}$, Kalb-Ramond field $B_{\mu \nu}$ and a dilaton field $\Phi$, described by the action $[3,4]$

$$
\begin{aligned}
S[x]= & \kappa \int_{\Sigma} \mathrm{d}^{2} \xi \sqrt{-g}\left[\left(\frac{1}{2} g^{\alpha \beta} G_{\mu \nu}(x)+\frac{\varepsilon^{\alpha \beta}}{\sqrt{-g}} B_{\mu \nu}(x)\right)\right. \\
& \left.\times \partial_{\alpha} x^{\mu} \partial_{\beta} x^{\nu}+\frac{1}{4 \pi \kappa} \Phi(x) R^{(2)}\right] .
\end{aligned}
$$

The integration goes over a 2-dimensional world-sheet $\Sigma$ parametrized by $\xi^{\alpha}\left(\xi^{0}=\tau, \xi^{1}=\sigma\right), g_{\alpha \beta}$ is the intrinsic world-sheet metric, $R^{(2)}$ corresponding 2-dimensional scalar curvature, $x^{\mu}(\xi), \mu=0,1, \ldots, D-1$ are the coordinates of the $D$-dimensional space-time, $\kappa=\frac{1}{2 \pi \alpha^{\prime}}$ with $\alpha^{\prime}$ being the Regge slope parameter and $\varepsilon^{01}=-1$.

\subsection{Weakly curved background}

The requirement of the quantum conformal invariance of the world-sheet results in the space-time equations of motion for the background fields. In the lowest order in the slope parameter $\alpha^{\prime}$ these equations are

$$
\begin{aligned}
& R_{\mu \nu}-\frac{1}{4} B_{\mu \rho \sigma} B_{\nu}{ }^{\rho \sigma}+2 D_{\mu} \partial_{\nu} \Phi=0, \\
& D_{\rho} B_{\mu \nu}^{\rho}-2 \partial_{\rho} \Phi B_{\mu \nu}^{\rho}=0, \\
& 4(\partial \Phi)^{2}-4 D_{\mu} \partial^{\mu} \Phi+\frac{1}{12} B_{\mu \nu \rho} B^{\mu \nu \rho} \\
& +4 \pi \kappa(D-26) / 3-R=0 .
\end{aligned}
$$


Here $B_{\mu \nu \rho}=\partial_{\mu} B_{\nu \rho}+\partial_{\nu} B_{\rho \mu}+\partial_{\rho} B_{\mu \nu}$ is the field strength of the field $B_{\mu \nu}$, and $R_{\mu \nu}$ and $D_{\mu}$ are the Ricci tensor and the covariant derivative with respect to the space-time metric. We will consider one of the simplest coordinate dependent solutions of (5), the weakly curved background. This background was considered in Refs. [21-23], where the influence of the boundary conditions on the non-commutativity of the open bosonic string has been investigated. The same approximation was considered in $[16,19]$ in context of the closed string non-commutativity.

The weakly curved background is defined by

$$
\begin{aligned}
G_{\mu \nu}(x) & =\text { const } \\
B_{\mu \nu}(x) & =b_{\mu \nu}+\frac{1}{3} B_{\mu \nu \rho} x^{\rho} \equiv b_{\mu \nu}+h_{\mu \nu}(x), \\
\Phi(x) & =\text { const }
\end{aligned}
$$

with $b_{\mu \nu}, B_{\mu \nu \rho}=$ const. This background is the solution of the space-time equations of motion if the constant $B_{\mu \nu \rho}$ is taken to be infinitesimal and all the calculations are done in the first order in $B_{\mu \nu \rho}$, so that the curvature $R_{\mu \nu}$ can be neglected as the infinitesimal of the second order. Through the whole manuscript (with the exception of Sect. 9) we assume that the background has the topology of $D$-dimensional torus $T^{D}$, where the Kalb-Ramond field depends on all coordinates. In Sects. 9.1 and 9.2 we give an example of the 3-dimensional torus, $T^{3}$, with H-flux, where the Kalb-Ramond field depends only on the coordinate $x^{3}$, while in Sect. 9.3 we give an example of the 4-dimensional torus $T^{4}$ with constant background fields.

The assumption that $B_{\mu \nu \rho}$ is infinitesimal means that we consider the $D$-dimensional torus so large that for any choice of indices

$\frac{B_{\mu v \rho}}{R_{\mu} R_{v} R_{\rho}} \ll 1$

holds [16], where $R_{\mu}$ are the radii of the torus. The $H$ flux background, considered in Refs. [8,16], is of the same type as the weakly curved background. However, this background depends just on $x^{3}$ and corresponds to the examples addressed in Sect. 9 of our paper. The background considered in the rest of the article depends on all coordinates.

In this paper we will investigate the T-dualization properties of the action (4) describing the closed string moving in the weakly curved background. Taking the conformal gauge $g_{\alpha \beta}=e^{2 F} \eta_{\alpha \beta}$, the action (4) becomes

$$
S[x]=\kappa \int_{\Sigma} \mathrm{d}^{2} \xi \partial_{+} x^{\mu} \Pi_{+\mu \nu}(x) \partial_{-} x^{\nu},
$$

with the background field composition equal to

$$
\Pi_{ \pm \mu \nu}(x)=B_{\mu \nu}(x) \pm \frac{1}{2} G_{\mu \nu}(x)
$$

and the light-cone coordinates given by

$\xi^{ \pm}=\frac{1}{2}(\tau \pm \sigma), \quad \partial_{ \pm}=\partial_{\tau} \pm \partial_{\sigma}$

\subsection{Complete T-dualization}

The T-dualization of the closed string theory in the weakly curved background was presented in [18]. The procedure is related to a global symmetry of the theory

$\delta x^{\mu}=\lambda^{\mu}$

The symmetry still exists in the presence of the nontrivial Kalb-Ramond field (6), but only in the case of the trivial mapping of the world-sheet into the space-time, because in that case the variation of the action (8)

$\delta S=\frac{\kappa}{3} \varepsilon^{\alpha \beta} B_{\mu \nu \rho} \lambda^{\rho} \int \mathrm{d}^{2} \xi \partial_{\alpha} x^{\mu} \partial_{\beta} x^{\nu}$

after partial integration, using the identity $\varepsilon^{\alpha \beta} \partial_{\alpha} \partial_{\beta}=0$, becomes

$\delta S=\frac{\kappa}{3} B_{\mu \nu \rho} \lambda^{\rho} \epsilon^{\alpha \beta} \int \mathrm{d}^{2} \xi \partial_{\alpha}\left(x^{\mu} \partial_{\beta} x^{\nu}\right)$,

which is equal to zero. This means that classically, directions which appear in the argument of Kalb-Ramond field are also Killing directions. However, the standard Buscher procedure cannot be applied to them, because background fields depend on the coordinates but not on their derivatives.

The T-dual picture of the theory, obtained on applying the T-dualization procedure to all the coordinates, is given by

$$
\begin{aligned}
S[y] & =\kappa \int \mathrm{d}^{2} \xi \partial_{+} y_{\mu}^{\star} \Pi_{+}^{\mu \nu}(\Delta V(y)) \partial_{-} y_{v} \\
& =\frac{\kappa^{2}}{2} \int \mathrm{d}^{2} \xi \partial_{+} y_{\mu} \Theta_{-}^{\mu \nu}(\Delta V(y)) \partial_{-} y_{\nu},
\end{aligned}
$$

where

$\Theta_{ \pm}^{\mu \nu} \equiv-\frac{2}{\kappa}\left(G_{E}^{-1} \Pi_{ \pm} G^{-1}\right)^{\mu \nu}=\theta^{\mu \nu} \mp \frac{1}{\kappa}\left(G_{E}^{-1}\right)^{\mu \nu}$,

with

$$
\begin{aligned}
G_{E \mu \nu} & \equiv G_{\mu \nu}-4\left(B G^{-1} B\right)_{\mu \nu}, \\
\theta^{\mu \nu} & \equiv-\frac{2}{\kappa}\left(G_{E}^{-1} B G^{-1}\right)^{\mu \nu},
\end{aligned}
$$

being the effective metric and the non-commutativity parameter in Seiberg-Witten terminology of the open bosonic string theory [24]. The T-dual background fields are equal to

$$
\begin{aligned}
{ }^{\star} G^{\mu \nu}(\Delta V(y)) & =\left(G_{E}^{-1}\right)^{\mu \nu}(\Delta V(y)), \\
{ }^{\star} B^{\mu \nu}(\Delta V(y)) & =\frac{\kappa}{2} \theta^{\mu \nu}(\Delta V(y)),
\end{aligned}
$$


and their argument is given by

$$
\begin{aligned}
\Delta V^{\mu}(y)= & -\frac{\kappa}{2}\left(\Theta_{0-}^{\mu \nu}+\Theta_{0+}^{\mu \nu}\right) \Delta y_{v} \\
& +\frac{\kappa}{2}\left(\Theta_{0-}^{\mu \nu}-\Theta_{0+}^{\mu \nu}\right) \Delta \tilde{y}_{v} \\
= & -\kappa \theta_{0}^{\mu \nu} \Delta y_{v}+\left(g^{-1}\right)^{\mu \nu} \Delta \tilde{y}_{v} .
\end{aligned}
$$

Here $\Theta_{0 \pm}^{\mu \nu}$ is the zeroth order value of the field composition $\Theta_{ \pm}^{\mu \nu}$ defined in (15) and $g_{\mu \nu}=G_{\mu \nu}-4 b_{\mu \nu}^{2}$ and $\theta_{0}^{\mu \nu}=$ $-\frac{2}{\kappa}\left(g^{-1} b G^{-1}\right)^{\mu \nu}$ are the zeroth order values of the effective fields (16). The variable $\Delta \tilde{y}_{\mu}$ is the double of the dual variable $\Delta y_{\mu}=y_{\mu}(\xi)-y_{\mu}\left(\xi_{0}\right)$, defined as the following line integral:

$\Delta \tilde{y}_{\mu}=\int_{P}\left(\mathrm{~d} \tau y_{\mu}^{\prime}+\mathrm{d} \sigma \dot{y}_{\mu}\right)=\int_{P} \mathrm{~d} \xi^{\alpha} \varepsilon_{\alpha}^{\beta} \partial_{\beta} y_{\mu}$,

taken along the path $P$, from the point $\xi_{0}^{\alpha}\left(\tau_{0}, \sigma_{0}\right)$ to the point $\xi^{\alpha}(\tau, \sigma)$

The fact that we are working with the weakly curved background ensures that the T-dual background fields are the solution of the space-time equations (5). Because both dual metric ${ }^{\star} G^{\mu \nu}$ and dual Kalb-Ramond field ${ }^{\star} B^{\mu \nu}$ are linear in coordinates with infinitesimal coefficients, the dual Christoffel symbol ${ }^{\star} \Gamma_{\mu}^{\nu \rho}$ and dual field strength ${ }^{\star} B^{\mu \nu \rho}$ are constant and infinitesimal. In Eq. (114) of Sect. 8 we will show that T-dual dilaton field is ${ }^{\bullet} \Phi=\Phi-\ln \operatorname{det} \sqrt{2 \Pi_{+}}$, where $\Phi$ is constant and $\Pi_{+}$is linear in coordinates with infinitesimal coefficients. So, ${ }^{\bullet} \Phi$ is also linear in coordinates with infinitesimal coefficients, and $\partial_{\mu} \bullet \Phi$ is constant and infinitesimal. Consequently, $D_{\mu} \partial_{\nu} \bullet \Phi, \partial_{\rho} \bullet \Phi B^{\rho}{ }_{\mu \nu}$ and $\left(\partial_{\mu} \bullet \Phi\right)^{2}$ are infinitesimals of the second order. So, all T-dual space-time equations, for the metric, for the Kalb-Ramond field and for dilaton field, are infinitesimals of the second order and as such are neglected.

The initial theory (8) and its completely T-dual theory (14) are connected by the T-dual coordinate transformation laws (eq. (42) of Ref. [18])

$\partial_{ \pm} x^{\mu}=-\kappa \Theta^{\mu v}(\Delta V) \partial_{ \pm} y_{v} \mp 2 \kappa \Theta_{0 \pm}^{\mu v} \beta_{v}^{\mp}(V)$,

and its inverse (eq. (66) of Ref. [18])

$\partial_{ \pm} y_{\mu} \cong-2 \Pi_{\mp \mu \nu}(\Delta x) \partial_{ \pm} x^{\nu} \mp 2 \beta_{\mu}^{\mp}(x)$,

where $\beta_{\mu}^{ \pm}(x)=\mp \frac{1}{2} h_{\mu \nu}(x) \partial_{\mp} x^{\nu}$. It is shown that

$\mathcal{T}: S\left[x^{\mu}\right] \rightarrow S\left[y_{\mu}\right], \quad \tilde{\mathcal{T}}: S\left[y_{\mu}\right] \rightarrow S\left[x^{\mu}\right]$

and therefore

$\mathcal{T} \circ \widetilde{\mathcal{T}}=1$
3 T-dualization along arbitrary subset of coordinates $\mathcal{T}^{a}: S\left[x^{\mu}\right] \rightarrow S\left[x^{i}, y_{a}\right]$

In this section, we will learn what theory is obtained if one chooses to apply the T-dualization procedure to the action (8), along arbitrary $d$ coordinates $x^{a}, \mathcal{T}^{a}: S\left[x^{\mu}\right] \rightarrow S\left[x^{i}, y_{a}\right]$, with $\mathcal{T}^{a}=\circ_{n=1}^{d} T^{\mu_{n}}, \mu_{n} \in(0,1, \ldots, D-1)$.

The closed string action in the weakly curved background (6) has a global symmetry (11). One localizes the symmetry for the coordinates $x^{a}$, by introducing the gauge fields $v_{\alpha}^{a}$ and substituting the ordinary derivatives with the covariant derivatives

$\partial_{\alpha} x^{a} \rightarrow D_{\alpha} x^{a}=\partial_{\alpha} x^{a}+v_{\alpha}^{a}$

The covariant derivatives are invariant under standard gauge transformations

$\delta v_{\alpha}^{a}=-\partial_{\alpha} \lambda^{a}$

In the case of the weakly curved background, in order to obtain the gauge invariant action one should additionally substitute the coordinates $x^{a}$ in the argument of the background fields with their invariant extension, defined by

$$
\begin{aligned}
\Delta x_{\text {inv }}^{a} & \equiv \int_{P} \mathrm{~d} \xi^{\alpha} D_{\alpha} x^{a}=\int_{P}\left(\mathrm{~d} \xi^{+} D_{+} x^{a}+\mathrm{d} \xi^{-} D_{-} x^{a}\right) \\
& =x^{a}-x^{a}\left(\xi_{0}\right)+\Delta V^{a},
\end{aligned}
$$

where

$\Delta V^{a} \equiv \int_{P} \mathrm{~d} \xi^{\alpha} v_{\alpha}^{a}=\int_{P}\left(\mathrm{~d} \xi^{+} v_{+}^{a}+\mathrm{d} \xi^{-} v_{-}^{a}\right)$

To preserve the physical equivalence between the gauged and the original theory, one introduces the Lagrange multipliers $y_{a}$ and adds term $\frac{1}{2} y_{a} F_{+-}^{a}$ to the Lagrangian, which will force the field strength $F_{+-}^{a} \equiv \partial_{+} v_{-}^{a}-\partial_{-} v_{+}^{a}=-2 F_{01}^{a}$ to vanish. In this way, the gauge invariant action

$$
\begin{aligned}
S_{\text {inv }} & {\left[x^{\mu}, x_{\text {inv }}^{a}, y_{a}\right] } \\
= & \kappa \int \mathrm{d}^{2} \xi\left[\partial_{+} x^{i} \Pi_{+i j}\left(x^{i}, \Delta x_{\text {inv }}^{a}\right) \partial_{-} x^{j}\right. \\
& +\partial_{+} x^{i} \Pi_{+i a}\left(x^{i}, \Delta x_{\text {inv }}^{a}\right) D_{-} x^{a} \\
& +D_{+} x^{a} \Pi_{+a i}\left(x^{i}, \Delta x_{\text {inv }}^{a}\right) \partial_{-} x^{i} \\
& +D_{+} x^{a} \Pi_{+a b}\left(x^{i}, \Delta x_{\text {inv }}^{a}\right) D_{-} x^{b} \\
& \left.+\frac{1}{2}\left(v_{+}^{a} \partial_{-} y_{a}-v_{-}^{a} \partial_{+} y_{a}\right)\right]
\end{aligned}
$$

is obtained, where the last term is equal to $\frac{1}{2} y_{a} F_{+-}^{a}$ up to the total divergence. Now, we can fix the gauge taking $x^{a}(\xi)=$ $x^{a}\left(\xi_{0}\right)$ and obtain the gauge fixed action

$S_{\mathrm{fix}}\left[x^{i}, v_{ \pm}^{a}, y_{a}\right]$ 


$$
\begin{aligned}
= & \kappa \int \mathrm{d}^{2} \xi\left[\partial_{+} x^{i} \Pi_{+i j}\left(x^{i}, \Delta V^{a}\right) \partial_{-} x^{j}\right. \\
& +\partial_{+} x^{i} \Pi_{+i a}\left(x^{i}, \Delta V^{a}\right) v_{-}^{a}+v_{+}^{a} \Pi_{+a i}\left(x^{i}, \Delta V^{a}\right) \partial_{-} x^{i} \\
& \left.+v_{+}^{a} \Pi_{+a b}\left(x^{i}, \Delta V^{a}\right) v_{-}^{b}+\frac{1}{2}\left(v_{+}^{a} \partial_{-} y_{a}-v_{-}^{a} \partial_{+} y_{a}\right)\right]
\end{aligned}
$$

This action reduces to the initial one for the equations of motion obtained varying over the Lagrange multipliers. The T-dual action is obtained for the equations of motion for the gauge fields.

\subsection{Regaining the initial action}

Varying the gauge fixed action (29) over the Lagrange multipliers $y_{a}$ one obtains the equations of motion

$\partial_{+} v_{-}^{a}-\partial_{-} v_{+}^{a}=0$

which have the solution

$v_{ \pm}^{a}=\partial_{ \pm} x^{a}$

On this solution the background fields' argument $\Delta V^{a}$ defined in (27) is path independent and reduces to

$\Delta V^{a}(\xi)=x^{a}(\xi)-x^{a}\left(\xi_{0}\right)$.

The gauge fixed action (29) reduces to the initial action (8), but the background fields' argument is $\Delta V^{a}$ instead of $x^{i}$. However, the action (8) is invariant under the constant shift of coordinates, so shifting coordinates by $x^{a}\left(\xi_{0}\right)$ one obtains the exact form of the initial action.

\subsection{The T-dual action}

Using the equations of motion for the gauge fields, we eliminate them and obtain the T-dual action.

The equations of motion obtained varying the gauge fixed action (29) over the gauge fields $v_{ \pm}^{a}$ are

$$
\begin{aligned}
& \Pi_{ \pm a i}\left(x^{i}, \Delta V^{a}\right) \partial_{\mp} x^{i}+\Pi_{ \pm a b}\left(x^{i}, \Delta V^{a}\right) v_{\mp}^{b}+\frac{1}{2} \partial_{\mp} y_{a} \\
& \quad= \pm \beta_{a}^{ \pm}\left(x^{i}, V^{a}\right)
\end{aligned}
$$

where

$$
\begin{aligned}
\beta_{a}^{ \pm}\left(x^{i}, V^{a}\right)= & \mp \frac{1}{2}\left[h_{a i}\left(x^{i}\right) \partial_{\mp} x^{i}+h_{a b}\left(x^{i}\right) \partial_{\mp} V^{b}\right. \\
& \left.+h_{a i}\left(V^{a}\right) \partial_{\mp} x^{i}+h_{a b}\left(V^{a}\right) \partial_{\mp} V^{b}\right]
\end{aligned}
$$

is the contribution from the background fields' argument $\Delta V^{a}$, defined in a same way as in Ref. [18], by $\delta_{V} S_{\mathrm{fix}}=$ $-\kappa \int \mathrm{d}^{2} \xi\left(\beta_{a}^{+} \delta v_{+}^{a}+\beta_{a}^{-} \delta v_{-}^{a}\right)$. If the initial background $\Pi_{+\mu \nu}$ does not depend on the coordinates $x^{a}$, the corresponding beta functions are zero $\beta_{a}^{ \pm}=0$.

Multiplying Eq. (33) by $2 \kappa \tilde{\Theta}_{\mp}^{a b}$, defined in (A.7), the inverse of the background fields composition $\Pi_{ \pm a b}$, one obtains

$$
\begin{aligned}
v_{\mp}^{a}= & -2 \kappa \tilde{\Theta}_{\mp}^{a b}\left(x^{i}, \Delta V^{a}\right)\left[\Pi_{ \pm b i}\left(x^{i}, \Delta V^{a}\right) \partial_{\mp} x^{i}+\frac{1}{2} \partial_{\mp} y_{b}\right. \\
& \left.\mp \beta_{b}^{ \pm}\left(x^{i}, V^{a}\right)\right] .
\end{aligned}
$$

Substituting (35) into the action (29), we obtain the T-dual action

$$
\begin{aligned}
& S\left[x^{i}, y_{a}\right] \\
& =\kappa \int \mathrm{d}^{2} \xi\left[\partial_{+} x^{i} \bar{\Pi}_{+i j}\left(x^{i}, \Delta V^{a}\left(x^{i}, y_{a}\right)\right) \partial_{-} x^{j}\right. \\
& \quad-\kappa \partial_{+} x^{i} \Pi_{+i a}\left(x^{i}, \Delta V^{a}\left(x^{i}, y_{a}\right)\right) \\
& \quad \times \tilde{\Theta}_{-}^{a b}\left(x^{i}, \Delta V^{a}\left(x^{i}, y_{a}\right)\right) \partial_{-} y_{b} \\
& \quad+\kappa \partial_{+} y_{a} \tilde{\Theta}_{-}^{a b}\left(x^{i}, \Delta V^{a}\left(x^{i}, y_{a}\right)\right) \\
& \quad \times \Pi_{+b i}\left(x^{i}, \Delta V^{a}\left(x^{i}, y_{a}\right)\right) \partial_{-} x^{i} \\
& \left.\quad+\frac{\kappa}{2} \partial_{+} y_{a} \tilde{\Theta}_{-}^{a b}\left(x^{i}, \Delta V^{a}\left(x^{i}, y_{a}\right)\right) \partial_{-} y_{b}\right]
\end{aligned}
$$

where

$\bar{\Pi}_{+i j} \equiv \Pi_{+i j}-2 \kappa \Pi_{+i a} \tilde{\Theta}_{-}^{a b} \Pi_{+b j}$.

In order to find the explicit value of the background fields argument $\Delta V^{a}\left(x^{i}, y_{a}\right)$, it is enough to consider the zeroth order of the equations of motion for the gauge fields $v_{ \pm}^{a}$ (35)

$v_{ \pm}^{(0) a}=-2 \kappa \tilde{\Theta}_{0 \pm}^{a b}\left[\Pi_{0 \mp b i} \partial_{ \pm} x^{(0) i}+\frac{1}{2} \partial_{ \pm} y_{b}^{(0)}\right]$.

Here $\tilde{\Theta}_{0 \pm}^{a b}$ and $\Pi_{0 \mp b i}$ stand for the zeroth order values of $\tilde{\Theta}_{ \pm}^{a b}$ and $\Pi_{\mp b i}$, and they are defined in (A.11).

Substituting (38) into (27) we obtain

$$
\begin{aligned}
\Delta V^{(0) a}\left(x^{i}, y_{a}\right) \\
=-\kappa\left[\tilde{\Theta}_{0+}^{a b} \Pi_{0-b i}+\tilde{\Theta}_{0-}^{a b} \Pi_{0+b i}\right] \Delta x^{(0) i} \\
\quad-\kappa\left[\tilde{\Theta}_{0+}^{a b} \Pi_{0-b i}-\tilde{\Theta}_{0-}^{a b} \Pi_{0+b i}\right] \Delta \tilde{x}^{(0) i} \\
\quad-\frac{\kappa}{2}\left[\tilde{\Theta}_{0+}^{a b}+\tilde{\Theta}_{0-}^{a b}\right] \Delta y_{b}^{(0)}-\frac{\kappa}{2}\left[\tilde{\Theta}_{0+}^{a b}-\tilde{\Theta}_{0-}^{a b}\right] \Delta \tilde{y}_{b}^{(0)} .
\end{aligned}
$$

Here

$\Delta \tilde{y}_{a}^{(0)}=\int_{P}\left(\mathrm{~d} \tau y_{a}^{(0) \prime}+\mathrm{d} \sigma \dot{y}_{a}^{(0)}\right)$,

$\Delta \tilde{x}^{(0) i}=\int_{P}\left(\mathrm{~d} \tau x^{(0) \prime i}+\mathrm{d} \sigma \dot{x}^{(0) i}\right)$

are the variables T-dual to the coordinates $y_{a}$ and $x^{i}$ in the zeroth order in $B_{\mu \nu \rho}$, for $b_{\mu \nu}=0$, which we call the double variables. 
Thus, we obtain the explicit form of the T-dual action and conclude that it is given in terms of the original coordinates $x^{i}$ and the dual coordinates $y_{a}$ originating from the Lagrange multipliers. However, the background fields' argument depends not only on these variables but on their doubles as well. Because of this the theory is non-local as the double variables $\tilde{x}^{i}$ and $\tilde{y}_{a}$ are defined as line integrals.

The action (36) can be obtained from the initial action (8) under the following substitutions of the coordinate derivatives and the background fields:

$\partial_{ \pm} x^{i} \rightarrow \partial_{ \pm} x^{i}, \quad \partial_{ \pm} x^{a} \rightarrow \partial_{ \pm} y_{a}$,

$\Pi_{+i j} \rightarrow \Pi_{+i j}, \quad \Pi_{+i a} \rightarrow \cdot \Pi_{+i}^{a}$,

$\Pi_{+a i} \rightarrow \bullet \Pi_{+i}^{a}, \quad \Pi_{+a b} \rightarrow \bullet \Pi_{+}^{a b}$,

where the dual background fields are

$\bullet \Pi_{+i j}=\bar{\Pi}_{+i j}, \quad \cdot \Pi_{+i}^{a}=-\kappa \Pi_{+i b} \tilde{\Theta}_{-}^{b a}$,

$\bullet \Pi_{+i}^{a}=\kappa \tilde{\Theta}_{-}^{a b} \Pi_{+b i}, \quad \cdot \Pi_{+}^{a b}=\frac{\kappa}{2} \tilde{\Theta}_{-}^{a b}$,

with $\bar{\Pi}_{+i j}, \Pi_{+\mu \nu}$, and $\tilde{\Theta}_{-}^{a b}$ defined in (37), (9), and (A.7). The argument of all T-dual background fields is $\left[x^{i}, V^{a}\left(x^{i}, y_{a}\right)\right]$. According to (27) and (39), it is non-local and consequently non-geometric. Calculating the symmetric and antisymmetric part of the T-dual field compositions (43), we find that the T-dual metric and Kalb-Ramond field are equal to

$$
\begin{aligned}
& { }^{\bullet} G_{i j}=\bar{G}_{i j}=G_{i j}-G_{i a}\left(\tilde{G}_{E}^{-1}\right)^{a b} G_{b j} \\
& -2 \kappa\left(B_{i a} \tilde{\theta}^{a b} G_{b j}+G_{i a} \tilde{\theta}^{a b} B_{b j}\right)-4 B_{i a}\left(\tilde{G}_{E}^{-1}\right)^{a b} B_{b j}, \\
& \text { - } B_{i j}=\bar{B}_{i j}=B_{i j}-\frac{\kappa}{2} G_{i a} \tilde{\theta}^{a b} G_{b j}-B_{i a}\left(\tilde{G}_{E}^{-1}\right)^{a b} G_{b j} \\
& -G_{i a}\left(\tilde{G}_{E}^{-1}\right)^{a b} B_{b j}-2 \kappa B_{i a} \tilde{\theta}^{a b} B_{b j}, \\
& { }^{-} G^{a b}=\left(\tilde{G}_{E}^{-1}\right)^{a b}, \\
& \text { - } B^{a b}=\frac{\kappa}{2} \tilde{\theta}^{a b}, \\
& { }^{\bullet} G^{a}{ }_{i}=\kappa \tilde{\theta}^{a b} G_{b i}+2\left(\tilde{G}_{E}^{-1}\right)^{a b} B_{b i}, \\
& \text { • } B_{i}^{a}=\kappa \tilde{\theta}^{a b} B_{b i}+\frac{1}{2}\left(\tilde{G}_{E}^{-1}\right)^{a b} G_{b i},
\end{aligned}
$$

where $\tilde{G}_{E a b}$ and $\tilde{\theta}^{a b}$ are defined in (A.6) and (A.10). The T-dual background fields have the same form as in the flat background $[1,5,25]$ but in the present case fields $B_{\mu \nu}, \tilde{G}_{E}^{-1 a b}$ and $\tilde{\theta}^{a b}$ are coordinate dependent.

Comparing the solutions for the gauge fields (31) and (35), we obtain the T-dual coordinate transformation law

$$
\begin{aligned}
\partial_{\mp} x^{a} \cong & -2 \kappa \tilde{\Theta}_{\mp}^{a b}\left(x^{i}, \Delta V^{a}\left(x^{i}, y_{a}\right)\right) \\
& \times\left[\Pi_{ \pm b i}\left(x^{i}, \Delta V^{a}\left(x^{i}, y_{a}\right)\right) \partial_{\mp} x^{i}+\frac{1}{2} \partial_{\mp} y_{b}\right. \\
& \left.\mp \beta_{b}^{ \pm}\left(x^{i}, V^{a}\left(x^{i}, y_{a}\right)\right)\right] .
\end{aligned}
$$

\section{Inverse T-dualization $\mathcal{T}_{a}: S\left[x^{i}, y_{a}\right] \rightarrow S\left[x^{\mu}\right]$}

In this section we will show that T-dualization of the action $S\left[x^{i}, y_{a}\right]$, given by (36), along already treated directions $y_{a}$ leads to the original action.

So, let us localize the global symmetry of the coordinates $y_{a}$

$\delta y_{a}=\lambda_{a}$,

of the action (36). Note that this is the symmetry, despite the coordinate dependence of the metric (44), due to the invariance of the background fields' argument [18]. Following the T-dualization procedure, we substitute the ordinary derivatives with the covariant ones

$D_{ \pm} y_{a}=\partial_{ \pm} y_{a}+u_{ \pm a}$,

where $u_{ \pm a}$ are gauge fields which transform as $\delta u_{ \pm a}=$ $-\partial_{ \pm} \lambda_{a}$. We also substitute coordinates $y_{a}$ in the background fields' argument with the invariant coordinates

$$
\begin{aligned}
y_{a}^{\mathrm{inv}} & =\int_{P}\left(\mathrm{~d} \xi^{+} D_{+} y_{a}+\mathrm{d} \xi^{-} D_{-} y_{a}\right) \\
& =y_{a}(\xi)-y_{a}\left(\xi_{0}\right)+\Delta U_{a},
\end{aligned}
$$

where

$\Delta U_{a}=\int_{P}\left(\mathrm{~d} \xi^{+} u_{+a}+\mathrm{d} \xi^{-} u_{-a}\right)$.

In this way, adding the Lagrange multiplier term which makes the introduced gauge fields nonphysical, we obtain the gauge invariant action

$$
\begin{aligned}
S_{\text {inv }} & {\left[x^{i}, y_{a}, y_{a}^{\text {inv }}, z^{a}\right] } \\
= & \kappa \int \mathrm{d}^{2} \xi\left[\partial_{+} x^{i} \bar{\Pi}_{+i j}\left(x^{i}, \Delta V^{a}\left(x^{i}, y_{a}^{\text {inv }}\right)\right) \partial_{-} x^{j}\right. \\
& -\kappa \partial_{+} x^{i} \Pi_{+i a}\left(x^{i}, \Delta V^{a}\left(x^{i}, y_{a}^{\text {inv }}\right)\right) \\
& \times \tilde{\Theta}_{-}^{a b}\left(x^{i}, \Delta V^{a}\left(x^{i}, y_{a}^{\text {inv }}\right)\right) D_{-} y_{b} \\
& +\kappa D_{+} y_{a} \tilde{\Theta}_{-}^{a b}\left(x^{i}, \Delta V^{a}\left(x^{i}, y_{a}^{\text {inv }}\right)\right) \\
& \times \Pi_{+b i}\left(x^{i}, \Delta V^{a}\left(x^{i}, y_{a}^{\text {inv }}\right)\right) \partial_{-} x^{i} \\
& +\frac{\kappa}{2} D_{+} y_{a} \tilde{\Theta}_{-}^{a b}\left(x^{i}, \Delta V^{a}\left(x^{i}, y_{a}^{\text {inv }}\right)\right) D_{-} y_{b} \\
& \left.+\frac{1}{2}\left(u_{+a} \partial_{-} z^{a}-u_{-a} \partial_{+} z^{a}\right)\right]
\end{aligned}
$$

which after fixing the gauge by $y_{a}(\xi)=y_{a}\left(\xi_{0}\right)$ becomes

$$
\begin{aligned}
S_{\mathrm{fix}} & {\left[x^{i}, u_{ \pm a}, z^{a}\right] } \\
= & \kappa \int \mathrm{d}^{2} \xi\left[\partial_{+} x^{i} \bar{\Pi}_{+i j}\left(x^{i}, \Delta V^{a}\left(x^{i}, \Delta U_{a}\right)\right) \partial_{-} x^{j}\right. \\
& -\kappa \partial_{+} x^{i} \Pi_{+i a}\left(x^{i}, \Delta V^{a}\left(x^{i}, \Delta U_{a}\right)\right) \\
& \times \tilde{\Theta}_{-}^{a b}\left(x^{i}, \Delta V^{a}\left(x^{i}, \Delta U_{a}\right)\right) u_{-b}
\end{aligned}
$$




$$
\begin{aligned}
& +\kappa u_{+a} \tilde{\Theta}_{-}^{a b}\left(x^{i}, \Delta V^{a}\left(x^{i}, \Delta U_{a}\right)\right) \\
& \times \Pi_{+b i}\left(x^{i}, \Delta V^{a}\left(x^{i}, \Delta U_{a}\right)\right) \partial_{-} x^{i} \\
& +\frac{\kappa}{2} u_{+a} \tilde{\Theta}_{-}^{a b}\left(x^{i}, \Delta V^{a}\left(x^{i}, \Delta U_{a}\right)\right) u_{-b} \\
& \left.+\frac{1}{2}\left(u_{+a} \partial_{-} z^{a}-u_{-a} \partial_{+} z^{a}\right)\right],
\end{aligned}
$$

where $\Delta V^{a}$ is defined in (39) and $\Delta U_{a}$ in (49).

\subsection{Regaining the T-dual action}

The equations of motion obtained varying the gauge fixed action (51) over the Lagrange multipliers $z^{a}$

$\partial_{+} u_{-a}-\partial_{-} u_{+a}=0$

have the solution

$u_{ \pm a}=\partial_{ \pm} y_{a}$

On this solution the variable $\Delta U_{a}$ defined by (49) is path independent and reduces to

$\Delta U_{a}(\xi)=y_{a}(\xi)-y_{a}\left(\xi_{0}\right)$,

and the gauge fixed action (51) reduces to the action (36).

\subsection{Regaining the initial action}

The equations of motion obtained varying the gauge fixed action (51) over the gauge fields $u_{ \pm a}$ are

$$
\begin{aligned}
\kappa & \tilde{\Theta}_{\mp}^{a b}\left(x^{i}, \Delta V^{a}\left(x^{i}, \Delta U_{a}\right)\right) \\
& \times\left[\frac{1}{2} u_{\mp b}+\Pi_{ \pm b i}\left(x^{i}, \Delta V^{a}\left(x^{i}, \Delta U_{a}\right)\right) \partial_{\mp} x^{i}\right]+\frac{1}{2} \partial_{\mp} z^{a} \\
= & \pm \kappa \tilde{\Theta}_{0 \mp}^{a b} \beta_{b}^{ \pm}\left(x^{i}, V^{a}\left(x^{i}, U_{a}\right)\right)
\end{aligned}
$$

where terms $\tilde{\Theta}_{0 \mp}^{a b} \beta_{b}^{ \pm}$are the contribution from the variation over the background field argument

$\delta_{U} S_{\mathrm{fix}}=-\kappa^{2} \int \mathrm{d}^{2} \xi\left(\delta u_{+a} \tilde{\Theta}_{0-}^{a b} \beta_{b}^{+}+\delta u_{-a} \tilde{\Theta}_{0+}^{a b} \beta_{b}^{-}\right)$.

Here $\beta_{a}^{ \pm}$is of the same form as (34) and $\tilde{\Theta}_{0 \mp}^{a b}$ is defined in (A.11).

Let us show that for the equations of motion (55), the gauge fixed action (51) will reduce to the initial action (8). Using the fact that $\tilde{\Theta}_{\mp}^{a b}$ is inverse to $2 \kappa \Pi_{ \pm a b}$, these equations of motion can be rewritten as

$$
\begin{aligned}
u_{\mp a}= & -2 \Pi_{ \pm a i}\left(x^{i}, \Delta V^{a}\left(x^{i}, \Delta U_{a}\right)\right) \partial_{\mp} x^{i} \\
& -2 \Pi_{ \pm a b}\left(x^{i}, \Delta V^{a}\left(x^{i}, \Delta U_{a}\right)\right) \partial_{\mp} z^{b} \\
& \pm 2 \beta_{a}^{ \pm}\left(x^{i}, V^{a}\left(x^{i}, U_{a}\right)\right) .
\end{aligned}
$$

Substituting (57) into (51), using the definition (37) and the first relation in (A.22) one obtains

$$
\begin{aligned}
& S\left[x^{i}, z^{a}\right] \\
& =\kappa \int_{\Sigma} \mathrm{d}^{2} \xi\left[\partial_{+} x^{i} \Pi_{+i j} \partial_{-} x^{j}+\partial_{+} x^{i} \Pi_{+i a} \partial_{-} z^{a}\right. \\
& \left.\quad+\partial_{+} z^{a} \Pi_{+a i} \partial_{-} x^{i}+\partial_{+} z^{a} \Pi_{+a b} \partial_{-} z^{b}\right] .
\end{aligned}
$$

The explicit form of the argument of the background fields is obtained substituting the zeroth order of Eq. (57) into (49)

$U_{a}^{(0)}=-2 b_{a i} x^{(0) i}+G_{a i} \tilde{x}^{(0) i}-2 b_{a b} z^{(0) b}+G_{a b} \tilde{z}^{(0) b}$.

Consequently, the argument of the background fields $\Delta V^{a}$, defined in (39), is just

$V^{(0) a}\left(x^{i}, U_{a}\right)=z^{a}$.

So, the action (58) is equal to the initial action (8) with $x^{\mu}=$ $\left(x^{i}, z^{a}\right)$.

Comparing the solutions for the gauge fields (53) and (57), we obtain the T-dual transformation law

$$
\begin{aligned}
\partial_{\mp} y_{a} \cong & -2 \Pi_{ \pm a i}\left(x^{i}, z^{a}\right) \partial_{\mp} x^{i}-2 \Pi_{ \pm a b}\left(x^{i}, z^{a}\right) \partial_{\mp} z^{b} \\
& \pm 2 \beta_{a}^{ \pm}\left(x^{i}, z^{a}\right) .
\end{aligned}
$$

Substituting $\partial_{\mp} y_{a}$ to (45) with the help of (60) one finds $\partial_{ \pm} x^{a}=\partial_{ \pm} z^{a}$. Therefore, (61) is the transformation inverse to (45), which confirms the relation $\mathcal{T}^{a} \circ \mathcal{T}_{a}=1$.

\section{T-dualization along all undualized coordinates $\mathcal{T}^{i}: S\left[x^{i}, y_{a}\right] \rightarrow S\left[y_{\mu}\right]$}

In this section we will T-dualize the action (36), applying the T-dualization procedure to the undualized coordinates $x^{i}$. Substituting the ordinary derivatives $\partial_{ \pm} x^{i}$ with the covariant derivatives

$D_{ \pm} x^{i}=\partial_{ \pm} x^{i}+w_{ \pm}^{i}$,

where the gauge fields $w_{ \pm}^{i}$ transform as $\delta w_{ \pm}^{i}=-\partial_{ \pm} \lambda^{i}$, substituting the coordinates $x^{i}$ in the background field arguments with

$\Delta x_{\mathrm{inv}}^{i}=\int_{P}\left(\mathrm{~d} \xi^{+} D_{+} x^{i}+\mathrm{d} \xi^{-} D_{-} x^{i}\right)$,

and adding the Lagrange multiplier term, we obtain the gauge invariant action

$$
\begin{aligned}
& S_{\mathrm{inv}}\left[x^{i}, x_{\mathrm{inv}}^{i}, y\right] \\
& =\kappa \int \mathrm{d}^{2} \xi\left[D_{+} x^{i} \bar{\Pi}_{+i j}\left(\Delta x_{\mathrm{inv}}^{i}, \Delta V^{a}\left(\Delta x_{\mathrm{inv}}^{i}, y_{a}\right)\right) D_{-} x^{j}\right. \\
& \quad-\kappa D_{+} x^{i} \Pi_{+i a}\left(\Delta x_{\mathrm{inv}}^{i}, \Delta V^{a}\left(\Delta x_{\mathrm{inv}}^{i}, y_{a}\right)\right)
\end{aligned}
$$




$$
\begin{aligned}
& \times \tilde{\Theta}_{-}^{a b}\left(\Delta x_{\mathrm{inv}}^{i}, \Delta V^{a}\left(\Delta x_{\mathrm{inv}}^{i}, y_{a}\right)\right) \partial_{-} y_{b} \\
& +\kappa \partial_{+} y_{a} \tilde{\Theta}_{-}^{a b}\left(\Delta x_{\mathrm{inv}}^{i}, \Delta V^{a}\left(\Delta x_{\mathrm{inv}}^{i}, y_{a}\right)\right) \\
& \times \Pi_{+b i}\left(\Delta x_{\mathrm{inv}}^{i}, \Delta V^{a}\left(\Delta x_{\mathrm{inv}}^{i}, y_{a}\right)\right) D_{-} x^{i} \\
& +\frac{\kappa}{2} \partial_{+} y_{a} \tilde{\Theta}_{-}^{a b}\left(\Delta x_{\mathrm{inv}}^{i}, \Delta V^{a}\left(\Delta x_{\mathrm{inv}}^{i}, y_{a}\right)\right) \partial_{-} y_{b} \\
& \left.+\frac{1}{2}\left(w_{+}^{i} \partial_{-} y_{i}-w_{-}^{i} \partial_{+} y_{i}\right)\right] .
\end{aligned}
$$

Substituting the gauge fixing condition $x^{i}(\xi)=x^{i}\left(\xi_{0}\right)$ one obtains

$$
\begin{aligned}
S_{\mathrm{fix}} & {\left[x^{i}, w_{ \pm}^{i}, y\right] } \\
= & \kappa \int \mathrm{d}^{2} \xi\left[w_{+}^{i} \bar{\Pi}_{+i j}(\Delta W) w_{-}^{j}\right. \\
& -\kappa w_{+}^{i} \Pi_{+i a}(\Delta W) \tilde{\Theta}_{-}^{a b}(\Delta W) \partial_{-} y_{b} \\
& +\kappa \partial_{+} y_{a} \tilde{\Theta}_{-}^{a b}(\Delta W) \Pi_{+b i}(\Delta W) w_{-}^{i} \\
& +\frac{\kappa}{2} \partial_{+} y_{a} \tilde{\Theta}_{-}^{a b}(\Delta W) \partial_{-} y_{b} \\
& \left.+\frac{1}{2}\left(w_{+}^{i} \partial_{-} y_{i}-w_{-}^{i} \partial_{+} y_{i}\right)\right],
\end{aligned}
$$

where $\Delta W^{\mu}=\left[\Delta W^{i}, \Delta V^{a}\left(\Delta W^{i}, y_{a}\right)\right]$ with $\Delta W^{i}$ defined by

$$
\Delta W^{i} \equiv \int_{P}\left(\mathrm{~d} \xi^{+} w_{+}^{i}+\mathrm{d} \xi^{-} w_{-}^{i}\right)
$$

and $\Delta V^{a}=\Delta V^{a}\left(\Delta W^{i}, y_{a}\right)$ is defined in (39), where argument $x^{i}$ is replaced by $\Delta W^{i}$.

\subsection{Regaining the T-dual action}

The equations of motion for the Lagrange multipliers $y_{i}$ are

$\partial_{+} w_{-}^{i}-\partial_{-} w_{+}^{i}=0$

and they have the solution

$w_{ \pm}^{i}=\partial_{ \pm} x^{i}$.

For this solution the background field argument $\Delta W^{i}$ defined in (66) reduces to

$\Delta W^{i}(\xi)=x^{i}(\xi)-x^{i}\left(\xi_{0}\right)$,

so that the argument $\Delta V^{a}$ becomes

$\Delta V^{a}\left(\Delta W^{i}, y^{a}\right)=\Delta V^{a}\left(x^{i}, y^{a}\right)$,

and therefore the gauge fixed action (65) reduces to the action (36).
5.2 From the gauge fixed action to the completely T-dual action

The equations of motion obtained varying the gauge fixed action (65) over $w_{ \pm}^{i}$ are

$$
\begin{aligned}
& \bar{\Pi}_{ \pm i j}(\Delta W) w_{\mp}^{j}-\kappa \Pi_{ \pm i a}(\Delta W) \tilde{\Theta}_{\mp}^{a b}(\Delta W) \partial_{\mp} y_{b}+\frac{1}{2} \partial_{\mp} y_{i} \\
& = \pm 2 \kappa \bar{\Pi}_{ \pm i j} \Theta_{\mp}^{j \mu} \beta_{\mu}^{ \pm}(W),
\end{aligned}
$$

where

$\beta_{\mu}^{ \pm}(V)=\mp \frac{1}{2} h_{\mu \nu}(V) \partial_{\mp} V^{\nu}$.

Terms $\bar{\Pi}_{ \pm i j} \Theta_{\mp}^{j \mu} \beta_{\mu}^{ \pm}(W)$ are the contribution from the background fields' argument, defined by

$$
\begin{array}{r}
\delta_{U} S_{\mathrm{fix}}=-2 \kappa^{2} \int \mathrm{d}^{2} \xi\left(\delta w_{+}^{i} \bar{\Pi}_{+i j} \Theta_{-}^{j \mu} \beta_{\mu}^{+}\right. \\
\left.+\delta w_{-}^{i} \bar{\Pi}_{-i j} \Theta_{+}^{j \mu} \beta_{\mu}^{-}\right),
\end{array}
$$

calculated using (A.15), (A.16), and (39).

Using the fact that the background field composition $\bar{\Pi}_{ \pm i j}$ is invese to $2 \kappa \Theta_{\mp}^{i j}$ defined by (A.22), we can rewrite the equation of motion (71) expressing the gauge fields as

$$
\begin{aligned}
w_{\mp}^{i}= & 2 \kappa \Theta_{\mp}^{i j}(\Delta W)\left[\kappa \Pi_{ \pm j a}(\Delta W) \tilde{\Theta}_{\mp}^{a b}(\Delta W) \partial_{\mp} y_{b}\right. \\
& \left.-\frac{1}{2} \partial_{\mp} y_{j}\right] \pm 2 \kappa \Theta_{0 \pm}^{i \mu} \beta_{\mu}^{ \pm}(W) .
\end{aligned}
$$

Using the second relation in (A.23), we obtain

$$
w_{\mp}^{i}=-\kappa \Theta_{\mp}^{i \mu}(\Delta W)\left[\partial_{\mp} y_{\mu} \mp 2 \beta_{\mu}^{ \pm}(W)\right] \text {. }
$$

Substituting (75) into the gauge fixed action (65), we obtain

$$
\begin{aligned}
S[y] & \\
= & \kappa \int \mathrm{d}^{2} \xi\left[\partial_{+} y_{i}\left(\kappa \Theta_{-}^{i j}-\kappa^{2} \Theta_{-}^{i k} \bar{\Pi}_{+k l} \Theta_{-}^{l j}\right) \partial_{-} y_{j}\right. \\
& +\left(-\kappa^{2} \Theta_{-}^{i j} \bar{\Pi}_{+j k} \Theta_{-}^{k a}+\frac{\kappa}{2} \Theta_{-}^{i a}-\kappa^{2} \Theta_{-}^{i j} \Pi_{+j b} \tilde{\Theta}_{-}^{b a}\right) \\
& \times \partial_{+} y_{i} \partial_{-} y_{a} \\
& +\left(-\kappa^{2} \Theta_{-}^{a j} \bar{\Pi}_{+j k} \Theta_{-}^{k i}+\frac{\kappa}{2} \Theta_{-}^{a i}-\kappa^{2} \tilde{\Theta}_{-}^{a b} \Pi_{+b j} \Theta_{-}^{j i}\right) \\
& \times \partial_{+} y_{a} \partial_{-} y_{i}+\partial_{+} y_{a}\left(\frac{\kappa}{2} \tilde{\Theta}_{-}^{a b}-\kappa^{2} \Theta_{-}^{a i} \bar{\Pi}_{+i j} \Theta_{-}^{j b}\right. \\
& \left.\left.-\kappa^{2} \Theta_{-}^{a i} \Pi_{+i c} \tilde{\Theta}_{-}^{c b}-\kappa^{2} \tilde{\Theta}_{-}^{a c} \Pi_{+c i} \Theta_{-}^{i b}\right) \partial_{-} y_{b}\right] .
\end{aligned}
$$

Using (A.22), (A.27), and (A.29) one can rewrite this action as

$$
S[y]=\frac{\kappa^{2}}{2} \int \mathrm{d}^{2} \xi \partial_{+} y_{\mu} \Theta_{-}^{\mu \nu}(\Delta W) \partial_{-} y_{\nu} .
$$


In order to find the background fields' argument $\Delta W^{i}$, we consider the zeroth order of Eqs. (75), and we conclude that

$\Delta W^{i}=-\kappa \theta_{0}^{i \mu} \Delta y_{\mu}+\left(g^{-1}\right)^{i \mu} \Delta \tilde{y}_{\mu}$.

Using (A.28) and (A.23), we find that $\Delta V^{a}\left(\Delta W^{i}, y^{a}\right)$ defined in (39) equals

$\Delta V^{a}\left(\Delta W^{i}, y_{a}\right)=-\kappa \theta_{0}^{a \mu} \Delta y_{\mu}+\left(g^{-1}\right)^{a \mu} \Delta \tilde{y}_{\mu}$.

Therefore, we conclude that the background fields' argument is equal to (18), so that the action (77) is the completely T-dual action (14), which is in agreement with Ref. [18]. Comparing the solutions for the gauge fields (68) and (75), we obtain the T-dual transformation law

$\partial_{\mp} x^{i} \cong-\kappa \Theta_{\mp}^{i \mu}(\Delta V(y))\left[\partial_{\mp} y_{\mu} \mp 2 \beta_{\mu}^{ \pm}(V(y))\right]$.

One can verify that two successive T-duality transformations (45) and (80) correspond to the total T-duality transformation (20). Indeed, the relation (80) is just the $i$ th component of this transformation. Substituting $\partial_{ \pm} x^{i}$ from (80) into (45), using (A.25) and (A.29), we obtain

$\partial_{ \pm} x^{a}=-\kappa \Theta_{ \pm}^{a \mu}(\Delta V)\left[\partial_{ \pm} y_{\mu} \pm 2 \beta_{\mu}^{\mp}(V)\right]$,

which is just the $a$ th component of the complete T-duality transformation. So, we confirm that $\mathcal{T}^{a} \circ \mathcal{T}^{i}=\mathcal{T}$.

\section{Inverse T-dualization along arbitrary subset of the dual coordinates $\mathcal{T}_{i}: S\left[y_{\mu}\right] \rightarrow S\left[x^{i}, y_{a}\right]$}

Finally, in this section we will show that the T-dualization of the completely T-dual action (14), along arbitrary subset of the dual coordinates $y_{i}$ leads to T-dual action (36). So, let us start with the T-dual action

$S[y]=\frac{\kappa^{2}}{2} \int \mathrm{d}^{2} \xi \partial_{+} y_{\mu} \Theta_{-}^{\mu \nu}(\Delta V(y)) \partial_{-} y_{\nu}$,

which is globally invariant to the constant shift of coordinates $y_{\mu}$

$\delta y_{\mu}=\lambda_{\mu}$.

We localize this symmetry for the coordinates $y_{i}$ and obtain the locally invariant action

$$
\begin{aligned}
S_{\text {inv }} & {\left[y, y_{i}^{\text {inv }}, z^{i}\right] } \\
= & \frac{\kappa^{2}}{2} \int \mathrm{d}^{2} \xi\left[D_{+} y_{i} \Theta_{-}^{i j}\left(\Delta V\left(y_{i}^{\text {inv }}, y_{a}\right)\right) D_{-} y_{j}\right. \\
& +D_{+} y_{i} \Theta_{-}^{i a}\left(\Delta V\left(y_{i}^{\text {inv }}, y_{a}\right)\right) \partial_{-} y_{a} \\
& +\partial_{+} y_{a} \Theta_{-}^{a i}\left(\Delta V\left(y_{i}^{\text {inv }}, y_{a}\right)\right) D_{-} y_{i}
\end{aligned}
$$

$$
\begin{aligned}
& +\partial_{+} y_{a} \Theta_{-}^{a b}\left(\Delta V\left(y_{i}^{\text {inv }}, y_{a}\right)\right) \partial_{-} y_{b} \\
& \left.+\frac{1}{\kappa}\left(u_{+i} \partial_{-} z^{i}-u_{-i} \partial_{+} z^{i}\right)\right],
\end{aligned}
$$

where $D_{ \pm} y_{i}=\partial_{ \pm} y_{i}+u_{ \pm i}$ are the covariant derivatives. The gauge fields $u_{ \pm i}$ transform as $\delta u_{ \pm i}=-\partial_{ \pm} \lambda_{i}$ and the invariant coordinates are defined by $y_{i}^{\text {inv }}=\int_{P}\left(\mathrm{~d} \xi^{+} D_{+} y_{i}+\right.$ $\left.\mathrm{d} \xi^{-} D_{-} y_{i}\right)$. After fixing the gauge by $y_{i}(\xi)=y_{i}\left(\xi_{0}\right)$, the action becomes

$$
\begin{aligned}
S_{\mathrm{fix}} & {\left[y_{a}, u_{ \pm i}, z^{i}\right] } \\
= & \frac{\kappa^{2}}{2} \int \mathrm{d}^{2} \xi\left[u_{+i} \Theta_{-}^{i j}\left(\Delta V\left(\Delta U_{i}, y_{a}\right)\right) u_{-j}\right. \\
& +u_{+i} \Theta_{-}^{i a}\left(\Delta V\left(\Delta U_{i}, y_{a}\right)\right) \partial_{-} y_{a} \\
& +\partial_{+} y_{a} \Theta_{-}^{a i}\left(\Delta V\left(\Delta U_{i}, y_{a}\right)\right) u_{-i} \\
& +\partial_{+} y_{a} \Theta_{-}^{a b}\left(\Delta V\left(\Delta U_{i}, y_{a}\right)\right) \partial_{-} y_{b} \\
& \left.+\frac{1}{\kappa}\left(u_{+i} \partial_{-} z^{i}-u_{-i} \partial_{+} z^{i}\right)\right]
\end{aligned}
$$

where $\Delta U_{i}=\int_{P}\left(\mathrm{~d} \xi^{+} u_{+i}+\mathrm{d} \xi^{-} u_{-i}\right)$.

\subsection{Regaining the T-dual action}

The equations of motion obtained varying the gauge fixed action (84) over the Lagrange multipliers

$\partial_{+} u_{-i}-\partial_{-} u_{+i}=0$,

have the solution

$u_{ \pm i}=\partial_{ \pm} y_{i}$

On this solution the variable $\Delta U_{i}$ reduces to

$\Delta U_{i}(\xi)=y_{i}(\xi)-y_{i}\left(\xi_{0}\right)$,

and therefore

$\Delta V^{\mu}\left(\Delta U_{i}, y_{a}\right)=\Delta V^{\mu}(y)$.

So, the action (84) becomes the action (81).

\subsection{Obtaining the T-dual action}

The equations of motion obtained varying the action (84) over $u_{ \pm i}$ are

$$
\begin{aligned}
& \kappa \Theta_{\mp}^{i j}\left(\Delta V\left(\Delta U_{i}, y_{a}\right)\right) u_{\mp j}+\kappa \Theta_{\mp}^{i a}\left(\Delta V\left(\Delta U_{i}, y_{a}\right)\right) \partial_{\mp} y_{a} \\
& \quad+\partial_{\mp} z^{i}= \pm 2 \kappa \Theta_{0 \mp}^{i \mu} \beta_{\mu}^{ \pm}\left(V\left(U_{i}, y_{a}\right)\right),
\end{aligned}
$$

where $\beta_{\mu}^{ \pm}$are given by (72). The terms with beta function come from the variation over the argument $U_{i}$

$\delta_{U} S_{\mathrm{fix}}=-\kappa^{2} \int \mathrm{d}^{2} \xi\left(\delta u_{+i} \Theta_{0-}^{i \mu} \beta_{\mu}^{+}+\delta u_{-i} \Theta_{0+}^{i \mu} \beta_{\mu}^{-}\right)$, 
and are calculated using (A.15) and (18). Using the fact that $2 \kappa \bar{\Pi}_{\mp i j}$ is the inverse of $\Theta_{ \pm}^{i j}$, the equation (89) can be rewritten as

$$
\begin{aligned}
u_{\mp i}= & -2 \bar{\Pi}_{ \pm i j}\left(\Delta V\left(\Delta U_{i}, y_{a}\right)\right)\left[\kappa \Theta_{\mp}^{j a}\left(\Delta V\left(\Delta U_{i}, y_{a}\right)\right)\right. \\
& \left.\times \partial_{\mp} y_{a}+\partial_{\mp} z^{j} \mp 2 \kappa \Theta_{0 \mp}^{j \mu} \beta_{\mu}^{ \pm}\left(V\left(U_{i}, y_{a}\right)\right)\right] .
\end{aligned}
$$

Substituting (91) into the gauge fixed action (84), using (A.25) we obtain

$$
\begin{aligned}
& S\left[z^{i}, y_{a}\right] \\
& =\frac{\kappa^{2}}{2} \int \mathrm{d}^{2} \xi\left[\frac{2}{\kappa} \partial_{+} z^{i} \bar{\Pi}_{+i j} \partial_{-} z^{j}+2 \partial_{+} z^{i} \bar{\Pi}_{+i j} \Theta_{-}^{j b} \partial_{-} y_{b}\right. \\
& \left.\quad-2 \partial_{+} y_{a} \Theta_{-}^{a i} \bar{\Pi}_{+i j} \partial_{-} z^{j}+\partial_{+} y_{a} \tilde{\Theta}_{-}^{a b} \partial_{-} y_{b}\right]
\end{aligned}
$$

which with the help of (A.29) becomes

$$
\begin{aligned}
S\left[z^{i}, y_{a}\right]= & \frac{\kappa^{2}}{2} \int \mathrm{d}^{2} \xi\left[\frac{2}{\kappa} \partial_{+} z^{i} \bar{\Pi}_{+i j} \partial_{-} z^{j}\right. \\
& -2 \partial_{+} z^{i} \Pi_{+i a} \tilde{\Theta}_{-}^{a b} \partial_{-} y_{b}+2 \partial_{+} y_{a} \tilde{\Theta}_{-}^{a b} \Pi_{+b j} \partial_{-} z^{j} \\
& \left.+\partial_{+} y_{a} \tilde{\Theta}_{-}^{a b} \partial_{-} y_{b}\right] .
\end{aligned}
$$

In order to find the argument of the background fields $\Delta V\left(\Delta U_{i}, y_{a}\right)$, one considers the zeroth order of Eqs. (91) and obtains

$$
\begin{aligned}
\Delta U_{i}^{(0)}= & -\left[\bar{\Pi}_{0+i j}+\bar{\Pi}_{0-i j}\right] \Delta z^{(0) j} \\
& +\left[\bar{\Pi}_{0+i j}-\bar{\Pi}_{0-i j}\right] \Delta \tilde{z}^{(0) j} \\
& -\kappa\left[\bar{\Pi}_{0+i j} \Theta_{0-}^{j a}+\bar{\Pi}_{0-i j} \Theta_{0+}^{j a}\right] \Delta y_{a}^{(0)} \\
& +\kappa\left[\bar{\Pi}_{0+i j} \Theta_{0-}^{j a}-\bar{\Pi}_{0-i j} \Theta_{0+}^{j a}\right] \Delta \tilde{y}_{a}^{(0)},
\end{aligned}
$$

where the double variables are defined in analogy with (40). Substituting (94) into (18), we obtain

$$
\Delta V^{i}\left(\Delta U_{i}, y_{a}\right)=\Delta z^{i},
$$

and

$$
\begin{aligned}
\Delta V^{a}\left(\Delta U_{i}, y_{a}\right)= & -\kappa\left[\tilde{\Theta}_{0+}^{a b} \Pi_{0-b i}+\tilde{\Theta}_{0-}^{a b} \Pi_{0+b i}\right] \Delta z^{(0) i} \\
& -\kappa\left[\tilde{\Theta}_{0+}^{a b} \Pi_{0-b i}-\tilde{\Theta}_{0-}^{a b} \Pi_{0+b i}\right] \Delta \tilde{z}^{(0) i} \\
& -\frac{\kappa}{2}\left[\tilde{\Theta}_{0+}^{a b}+\tilde{\Theta}_{0-}^{a b}\right] \Delta y_{b}^{(0)} \\
& -\frac{\kappa}{2}\left[\tilde{\Theta}_{0+}^{a b}-\tilde{\Theta}_{0-}^{a b}\right] \Delta \tilde{y}_{b}^{(0)},
\end{aligned}
$$

which is exactly (39) with $z^{i}=x^{i}$. So, we can conclude that the action (93) is equal to the T-dual action (36).

Comparing the solutions for the gauge fields (86) and (91), we obtain the T-dual transformation law

$$
\begin{aligned}
\partial_{\mp} y_{i} \cong & -2 \bar{\Pi}_{ \pm i j}\left(\Delta z^{i}, \Delta V^{a}\left(\Delta U_{i}\left(z^{i}, y_{a}\right), y_{a}\right)\right) \\
& \times\left[\kappa \Theta_{\mp}^{j a}\left(\Delta z^{i}, \Delta V^{a}\left(\Delta U_{i}\left(z^{i}, y_{a}\right), y_{a}\right)\right) \partial_{\mp} y_{a}+\partial_{\mp} z^{j}\right. \\
& \left.\mp 2 \kappa \Theta_{0 \mp}^{j \mu} \beta_{\mu}^{ \pm}\left(z^{i}, V^{a}\left(U_{i}\left(z^{i}, y_{a}\right), y_{a}\right)\right)\right] .
\end{aligned}
$$

These transformations are inverse to (80), so that $\mathcal{T}^{i} \circ \mathcal{T}_{i}=$ 1. Successively applying (97) and (61), using (A.29) and (A.25), we obtain the $i$ th component of the inverse law of the total T-dualization (21). Its $a$ th component is (61), so we confirm that $\mathcal{T}_{a} \circ \mathcal{T}_{i}=\tilde{\mathcal{T}}$.

\section{Group of the T-dual transformation laws}

In this section we will recapitulate the coordinate transformation laws between the theories considered. In Sect. 3, we performed the T-dualization procedure along the coordinates $x^{a}$

$\mathcal{T}^{a}: S\left[x^{\mu}\right] \rightarrow S\left[x^{i}, y_{a}\right]$,

and obtained the following coordinate transformation law: (45)

$$
\begin{aligned}
\partial_{\mp} x^{a} \cong & -2 \kappa \tilde{\Theta}_{\mp}^{a b}\left(x^{i}, \Delta V^{a}\left(x^{i}, y_{a}\right)\right) \\
& \times\left[\Pi_{ \pm b i}\left(x^{i}, \Delta V^{a}\left(x^{i}, y_{a}\right)\right) \partial_{\mp} x^{i}+\frac{1}{2} \partial_{\mp} y_{b}\right. \\
& \left.\mp \beta_{b}^{ \pm}\left(x^{i}, V^{a}\left(x^{i}, y_{a}\right)\right)\right]
\end{aligned}
$$

where $V^{a}$ and $\beta_{a}^{ \pm}$are given by (39) and (34). In the zeroth oder this law implies

$x^{(0) a} \cong V^{(0) a}\left(x^{i}, y_{a}\right)$.

In Sect. 4, starting from the action $S\left[x^{i}, y_{a}\right]$ we performed the T-dualization procedure along the coordinates $y_{a}$

$\mathcal{T}_{a}: S\left[x^{i}, y_{a}\right] \rightarrow S\left[x^{\mu}\right]$,

and obtained the transformation law (61)

$\partial_{\mp} y_{a} \cong-2 \Pi_{ \pm a \mu}(x) \partial_{\mp} x^{\mu} \pm 2 \beta_{a}^{ \pm}(x)$,

which is the law inverse to (99) and in the zeroth order it implies

$y_{a}^{(0)} \cong U_{a}^{(0)}(x)$.

Multiplying the transformation law (99) from the left side by $\Pi_{ \pm c a}(x) \cong \Pi_{ \pm c a}\left(x^{i}, \Delta V^{a}\left(x^{i}, y_{a}\right)\right)$, using (100), we obtain the transformation law (102). So, we confirm that $\mathcal{T}^{a} \circ \mathcal{T}_{a}=1$. 
In Sect. 5, starting once again from the action $S\left[x^{i}, y_{a}\right]$, we performed the T-dualization procedure along the undualized coordinates $x^{i}$

$\mathcal{T}^{i}: S\left[x^{i}, y_{a}\right] \rightarrow S\left[y_{\mu}\right]$

and obtained the coordinate transformation law (80)

$\partial_{\mp} x^{i} \cong-\kappa \Theta_{\mp}^{i \mu}(\Delta V(y))\left[\partial_{\mp} y_{\mu} \mp 2 \beta_{\mu}^{ \pm}(V(y))\right]$,

where $V^{\mu}$ and $\beta_{\mu}^{ \pm}$are given by (18) and (72). In the zeroth order it gives

$x^{(0) i} \cong V^{(0) i}(y)$.

The two successive T-duality transformations (99) and (105) give the complete transformation (20), so that $\mathcal{T}^{a} \circ \mathcal{T}^{i}=\mathcal{T}$.

In Sect. 6, starting from the completely T-dual action $S[y]$, we performed the T-dualization procedure along the coordinates $y_{i}$

$\mathcal{T}_{i}: S\left[y_{\mu}\right] \rightarrow S\left[x^{i}, y_{a}\right]$

and obtained (97)

$$
\begin{aligned}
\partial_{\mp} y_{i} \cong & -2 \bar{\Pi}_{ \pm i j}\left(\Delta x^{i}, \Delta V^{a}\left(\Delta U_{i}\left(x^{i}, y_{a}\right), y_{a}\right)\right) \\
& \times\left[\kappa \Theta_{\mp}^{j a}\left(\Delta x^{i}, \Delta V^{a}\left(\Delta U_{i}\left(x^{i}, y_{a}\right), y_{a}\right)\right) \partial_{\mp} y_{a}+\partial_{\mp} x^{j}\right. \\
& \left.\mp 2 \kappa \Theta_{0 \mp}^{j \mu} \beta_{\mu}^{ \pm}\left(x^{i}, V^{a}\left(U_{i}\left(x^{i}, y_{a}\right), y_{a}\right)\right)\right]
\end{aligned}
$$

with $V^{a}, U_{i}$, and $\beta_{\mu}^{ \pm}$given by (79), (94), and (72). In the zeroth order this law implies

$y_{i}^{(0)} \cong U_{i}^{(0)}\left(x^{i}, y_{a}\right)$.

Multiplying (108) from the left by

$\Theta_{\mp}^{k i}\left(\Delta x^{i}, \Delta V^{a}(y)\right) \cong \Theta_{\mp}^{k i}\left(\Delta x^{i}, \Delta V^{a}\left(\Delta U_{i}\left(x^{i}, y_{a}\right), y_{a}\right)\right)$,

using (106), we obtain the transformation law (105), so that $\mathcal{T}^{i} \circ \mathcal{T}_{i}=1$. Successively applying (108) and (102), using (A.29) and (A.25), we obtain the $i$ th component of the inverse law of the complete T-dualization (21). Its $a$ th component is (102), so we confirm that $\mathcal{T}_{a} \circ \mathcal{T}_{i}=\tilde{\mathcal{T}}$.

We can conclude that the elements $1, \mathcal{T}^{a}$ and $\mathcal{T}_{a}$, with $d=1, \ldots, D$, form an Abelian group. The element $\mathcal{T}^{a}$ is the inverse of the element $\mathcal{T}_{a}$.

\section{Dilaton field in the weakly curved background}

The T-duality transformation of the dilaton field in the weakly curved background was considered in Ref. [26]. For completeness and further use, we give here a brief recapitulation of some basic steps of the treatment.
It is well known that a dilaton transformation has a quantum origin. So, let us start with the path integral for the gauge fixed action

$\mathcal{Z}=\int \mathrm{d} v_{+}^{\mu} \mathrm{d} v_{-}^{\mu} \mathrm{d} y_{\mu} e^{i S_{\mathrm{fix}}\left(v_{ \pm}, \partial_{ \pm} y\right)}$,

where

$S_{\mathrm{fix}}\left(v_{ \pm}, \partial_{ \pm} y\right)=S_{0}+S_{1}$

with $S_{1}$ being the infinitesimal part of the action

$S_{0}=\kappa \int \mathrm{d}^{2} \xi\left[v_{+}^{\mu} \Pi_{0+\mu \nu} v_{-}^{\nu}+\frac{1}{2}\left(v_{+}^{\mu} \partial_{-} y_{\mu}-v_{-}^{\mu} \partial_{+} y_{\mu}\right)\right]$,

$S_{1}=\kappa \int \mathrm{d}^{2} \xi v_{+}^{\mu} h_{\mu \nu}(V) v_{-}^{v}$.

For a constant background $\left(S_{1}=0\right)$ the path integral is Gaussian and it equals (det $\left.\Pi_{0+\mu \nu}\right)^{-1}$. In our case the background is coordinate dependent and thus the integral is not Gaussian. The fact that we work with an infinitesimal parameter enables us to show that the final result is formally the same as in the flat case [26],

$\mathcal{Z}=\int \mathrm{d} y_{\mu} \frac{1}{\operatorname{det}\left(\Pi_{+\mu \nu}(V)\right)} e^{i \star S(y)}$,

where ${ }^{\star} S(y)=\frac{\kappa^{2}}{2} \int \mathrm{d}^{2} \xi \partial_{+} y_{\mu} \Theta_{-}^{\mu \nu}(V) \partial_{-} y_{\nu}$ is the complete T-dual action and $\Pi_{+\mu \nu}(V)=B_{\mu \nu}(V)+\frac{1}{2} G_{\mu \nu}$. Consequently, although for the weakly curved background the functional integration over $v_{ \pm}$is of the third degree, it produces formally the same result as in the flat space (where the action is Gaussian),

$\bullet \Phi=\Phi-\ln \operatorname{det} \sqrt{2 \Pi_{+a b}}$.

Using the expressions for T-dual fields (43) we can find the relations between the determinants

$$
\begin{aligned}
\operatorname{det}\left(2 \Pi_{ \pm a b}\right) & =\frac{1}{\operatorname{det}\left(2 \bullet \Pi_{ \pm}^{a b}\right)}=\sqrt{\frac{\operatorname{det} G_{a b}}{\operatorname{det} \bullet G^{a b}}} \\
& =\sqrt{\frac{\operatorname{det} G_{\mu \nu}}{\operatorname{det} \bullet G_{\mu \nu}}},
\end{aligned}
$$

where because of the relation $\Pi_{ \pm a b}=B_{a b} \pm \frac{1}{2} G_{a b}$ we put in the factor 2 for convenience. The symbol ${ }^{\bullet} G_{\mu \nu}$ denotes metric in the whole space-time after partial T-dualization along $x^{a}$ directions. With the help of last relation we can show that the change of space-time measure in the path integral is correct

$$
\begin{aligned}
& \sqrt{\operatorname{det} G_{\mu \nu}} \mathrm{d} x^{i} \mathrm{~d} x^{a} \rightarrow \sqrt{\operatorname{det} G_{\mu \nu}} \mathrm{d} x^{i} \frac{1}{\operatorname{det}\left(2 \Pi_{+a b}\right)} \mathrm{d} y_{a} \\
& =\sqrt{\operatorname{det}^{\bullet} G_{\mu \nu}} \mathrm{d} x^{i} \mathrm{~d} y_{a},
\end{aligned}
$$

when we performed T-dualization $T^{a}$ along $x^{a}$ directions. 


\section{Comparison with the existing facts}

\subsection{T-dualization chain for the background with $H$ flux}

In this section we will compare our results with the T-dualization chain of Ref. [16]. The coordinates of the $D=3$ dimensional torus will be denoted by $x^{1}, x^{2}, x^{3}$. Because of the different notation, the background fields considered in this paper and those considered in [16], which will be denoted $\mathcal{G}$ and $\mathcal{B}$, are related by

$\mathcal{B}_{\mu \nu}=-2 B_{\mu \nu}, \quad \mathcal{G}_{\mu \nu}=G_{\mu \nu}, \quad \mu, \nu=1,2,3$.

Nontrivial components of the background considered in Ref. [16] are

$\mathcal{G}_{\mu \nu}=\delta_{\mu \nu}, \quad \mathcal{B}_{12}=H x^{3}$,

which in our notation corresponds to the background fields

$G_{\mu \nu}=\delta_{\mu \nu}, \quad B_{12}=-\frac{1}{2} H x^{3}$.

Let us first compare the results in the case $d=1$, corresponding to the transition

$T^{1}$ : torus with H-flux $\rightarrow$ twisted torus.

To do so, let us perform T-dualization along the direction $x^{1}, T^{1}: S[x] \rightarrow S\left[y_{1}, x^{2}, x^{3}\right]$, for the string moving in the background (119). The indices take the values $a, b \in\{1\}$ and $i, j \in\{2,3\}$. Because the only nontrivial component of the Kalb-Ramond field is $B_{a i}=-\frac{1}{2} H x^{3} \delta_{i 2}$, the effective fields are just $\tilde{G}_{\mu \nu}^{E}=\delta_{\mu \nu}$ and $\tilde{\theta}^{a b}=0$. So, the T-dual background fields (44), in the linear order in $H$, are

${ }^{\bullet} G_{i j}=\delta_{i j}, \quad{ }^{\bullet} B_{i j}=0$,

${ }^{\bullet} G^{a b}=\delta^{a b}, \quad B^{a b}=0$,

${ }^{\bullet} G^{a}{ }_{i}=-H x^{3} \delta_{i 2}, \quad{ }^{a} B_{i}=0$.

Therefore

${ }^{\bullet} G_{\mu \nu}=\left(\begin{array}{ccc}1 & -H x^{3} & 0 \\ -H x^{3} & 1 & 0 \\ 0 & 0 & 1\end{array}\right)={ }^{\circ} \mathcal{G}_{\mu \nu}$,

and

${ }^{\bullet} B_{\mu \nu}=0={ }^{\bullet} \mathcal{B}_{\mu \nu}$,

so our result is in agreement with that of Ref. [16].

Now, let us make the comparison in the case $d=2$, which corresponds to the transition

$T^{1} \circ T^{2}$ : torus with H-flux $\rightarrow$ Q-flux non-geometry.
Instead to perform $T^{2}$ dualization, from twisted torus to $Q$ flux non-geometry as in [16], we will start from the initial background with $H$-flux and perform T-dualizations along $x^{1}$ and $x^{2}, T^{1} \circ T^{2}: S[x] \rightarrow S\left[y_{1}, y_{2}, x^{3}\right]$. The indices take the values $a, b \in\{1,2\}$ and $i, j \in\{3\}$. Because the only nontrivial contribution to the Kalb-Ramond field $B_{a b}$ is $B_{12}=-\frac{1}{2} H x^{3}$, the effective background fields are $\tilde{G}_{a b}^{E}=$ $\delta_{a b}, \bar{G}_{i j}^{E}=\delta_{i j}$, and the only nonzero component of $\tilde{\theta}^{a b}$ is $\tilde{\theta}^{12}=\frac{1}{\kappa} H x^{3}$. The T-dual background fields linear in $H$ are therefore

${ }^{\bullet} G_{i j}=\delta_{i j}, \quad{ }^{\bullet} G^{a b}=\delta^{a b}, \quad{ }^{\bullet} G^{a} i=0$,

and

${ }^{\bullet} B_{i j}=0, \quad{ }^{\prime} B^{12}=\frac{1}{2} H x^{3}, \quad{ }^{\bullet} B^{a}{ }_{i}=0$.

Consequently

${ }^{\bullet} G_{\mu \nu}=\left(\begin{array}{ccc}1 & 0 & 0 \\ 0 & 1 & 0 \\ 0 & 0 & 1\end{array}\right)={ }^{\bullet} \mathcal{G}_{\mu \nu}$,

${ }^{-} \mathcal{B}_{\mu \nu}=-2 \cdot B_{\mu \nu}=\left(\begin{array}{ccc}0 & -H x^{3} & 0 \\ H x^{3} & 0 & 0 \\ 0 & 0 & 0\end{array}\right)$,

so the results of this paper and [16] in this case coincide.

9.2 Non-associativity of R-flux background and breaking of Jacobi identity

In Refs. [18, 19] we obtained T-dual transformation laws connecting T-dual coordinates $y_{\mu}$ with the initial coordinates $x^{\mu}$. Here we will reduce our case to the 3 -dimensional torus with H-flux considered in [8]. Then, the full T-dualization along all coordinates corresponds to the so-called R-flux. So, we are going to calculate its characteristic features: nonassociativity relation and breaking of Jacobi identity.

We will work in the background of Sect. 9.1 consisting of euclidean flat metric $G_{\mu \nu}$ and Kalb-Ramond field with one nontrivial component $B_{12}=-\frac{1}{2} H x^{3}$. T-dual transformation laws for coordinates $y_{\mu}(\mu=1,2,3)$ are of the form

$y_{1}^{\prime} \cong \frac{1}{\kappa} \pi_{1}+\frac{1}{2} H x^{3} x^{\prime 2}$,

$y_{2}^{\prime} \cong \frac{1}{\kappa} \pi_{2}-\frac{1}{2} H x^{3} x^{\prime 1}$,

$y_{3}^{\prime} \cong \frac{1}{\kappa} \pi_{3}$,

where $\pi_{1}, \pi_{2}, \pi_{3}$ are canonically conjugated momenta for coordinates $x^{1}, x^{2}, x^{3}$, respectively. The initial space is a geometric one, so, the standard Poisson algebra is satisfied, 
$\left\{x^{\mu}(\sigma), \pi_{v}(\bar{\sigma})\right\}=\delta^{\mu}{ }_{\nu} \delta(\sigma-\bar{\sigma})$,

$\left\{x^{\mu}, x^{\nu}\right\}=\left\{\pi_{\mu}, \pi_{\nu}\right\}=0$.

From (127)-(129) we obtain

$\left\{y_{\mu}^{\prime}(\sigma), y_{\nu}^{\prime}(\bar{\sigma})\right\}=-\frac{1}{2 \kappa} H \varepsilon_{\mu \nu \rho} x^{\prime \rho} \delta(\sigma-\bar{\sigma})$,

which, after two partial integrations, produces

$\left\{y_{\mu}(\sigma), y_{\nu}(\bar{\sigma})\right\}=\frac{1}{2 \kappa} H \varepsilon_{\mu \nu \rho}\left[x^{\rho}(\sigma)-x^{\rho}(\bar{\sigma})\right] \theta(\sigma-\bar{\sigma})$,

where $\varepsilon_{\mu \nu \rho}$ is the 3-dimensional Levi-Civita tensor $\left(\varepsilon_{123}=\right.$ 1 ) and the function $\theta(\sigma)$ is defined as

$\theta(\sigma) \equiv \begin{cases}0 & \text { if } \sigma=0 \\ 1 / 2 & \text { if } 0<\sigma<2 \pi, \quad \sigma \in[0,2 \pi] . \\ 1 & \text { if } \sigma=2 \pi\end{cases}$

Using the standard Poisson algebra (130) and transformation laws (127)-(129), after one partial integration, we get

$$
\begin{aligned}
& \left\{\left\{y_{\mu}\left(\sigma_{1}\right), y_{v}\left(\sigma_{2}\right)\right\}, y_{\rho}\left(\sigma_{3}\right)\right\} \\
& =\frac{1}{2 \kappa^{2}} H \varepsilon_{\mu \nu \rho}\left[\theta\left(\sigma_{2}-\sigma_{1}\right) \theta\left(\sigma_{1}-\sigma_{3}\right)\right. \\
& \left.\quad+\theta\left(\sigma_{1}-\sigma_{2}\right) \theta\left(\sigma_{2}-\sigma_{3}\right)\right]
\end{aligned}
$$

Now we have all ingredients to calculate the nonassociativity relation

$$
\begin{aligned}
& \left\{\left\{y_{\mu}\left(\sigma_{1}\right), y_{\nu}\left(\sigma_{2}\right)\right\}, y_{\rho}\left(\sigma_{3}\right)\right\}-\left\{y_{\mu}\left(\sigma_{1}\right),\left\{y_{\nu}\left(\sigma_{2}\right), y_{\rho}\left(\sigma_{3}\right)\right\}\right\} \\
& =\frac{1}{2 \kappa^{2}} H \varepsilon_{\mu \nu \rho}\left[2 \theta\left(\sigma_{3}-\sigma_{2}\right) \theta\left(\sigma_{2}-\sigma_{1}\right)\right. \\
& \left.\quad+\theta\left(\sigma_{1}-\sigma_{3}\right) \theta\left(\sigma_{3}-\sigma_{2}\right)+\theta\left(\sigma_{3}-\sigma_{1}\right) \theta\left(\sigma_{1}-\sigma_{2}\right)\right]
\end{aligned}
$$

and breaking of Jacobi identity

$$
\begin{aligned}
&\left\{y_{\mu}\left(\sigma_{1}\right), y_{v}\left(\sigma_{2}\right), y_{\rho}\left(\sigma_{3}\right)\right\} \\
& \equiv\left\{\left\{y_{\mu}\left(\sigma_{1}\right), y_{\nu}\left(\sigma_{2}\right)\right\}, y_{\rho}\left(\sigma_{3}\right)\right\}+\left\{\left\{y_{v}\left(\sigma_{2}\right), y_{\rho}\left(\sigma_{3}\right)\right\}, y_{\mu}\left(\sigma_{1}\right)\right\} \\
& \quad+\left\{\left\{y_{\rho}\left(\sigma_{3}\right), y_{\mu}\left(\sigma_{1}\right)\right\}, y_{v}\left(\sigma_{2}\right)\right\} \\
&=\frac{1}{\kappa^{2}} H \varepsilon_{\mu \nu \rho}\left[\theta\left(\sigma_{1}-\sigma_{2}\right) \theta\left(\sigma_{2}-\sigma_{3}\right)\right. \\
&\left.\quad+\theta\left(\sigma_{3}-\sigma_{1}\right) \theta\left(\sigma_{1}-\sigma_{2}\right)+\theta\left(\sigma_{2}-\sigma_{3}\right) \theta\left(\sigma_{3}-\sigma_{1}\right)\right] .
\end{aligned}
$$

For example, for $\sigma_{1}=2 \pi+\sigma$ and $\sigma_{2}=\sigma_{3}=\sigma$ one has

$$
\left\{y_{\mu}(2 \pi+\sigma), y_{\nu}(\sigma), y_{\rho}(\sigma)\right\}=-\frac{1}{\kappa^{2}} H \varepsilon_{\mu \nu \rho} .
$$

In the approach of this article, the background of the $\mathrm{T}$ dual theory depends on the non-local variable $V^{\mu}$, which incorporates the main features of the non-geometric spaces.
Reducing our procedure to three dimensions and using the backgrounds of Refs. [8, 16,27], we showed that our structure of arguments of background fields proves the proposal of Refs. [8,27] that non-associativity and breaking of Jacobi identity are features of R-flux background.

\subsection{Critical surface}

Let us generalize the discussion of Ref. [20] where the critical surface, which separates equivalent sections of background fields, generalizes the critical radius. Using the dilaton field analysis, namely the relation (115), we can conclude that T-duality maps the theories with a given

$\operatorname{det}\left(2 \Pi_{ \pm a b}\right)$

into the theories with

$1 / \operatorname{det}\left(2 \Pi_{ \pm a b}\right)$

so that all different theories are in the region

$\operatorname{det}\left(2 \Pi_{ \pm a b}\right) \leq 0$

The theories which background fields satisfy the condition $\operatorname{det}\left(2 \Pi_{ \pm a b}\right)=1$, are mapped into each other under $\mathrm{T}$ duality. This is a generalization of the critical radius and can be consider as a critical surface. So, relation (115) implies $\sqrt{\operatorname{det} G_{a b}}=\sqrt{\operatorname{det}^{\bullet} G^{a b}}$, which means that a dual volume is equal to the initial one. At the critical surface the extended symmetry should be expected.

Let us, following [20], give an example of the relation between the original and T-dual background fields. We will consider the initial background in the 4-dimensional torus $T^{4}$ given by

$G_{\mu \nu}=g \delta_{\mu \nu}, \quad B_{\mu \nu}=b^{i} E_{\mu \nu}^{i}$,

where

$$
\begin{aligned}
E^{1}= & {\left[\begin{array}{cccc}
0 & 0 & 0 & 1 \\
0 & 0 & 1 & 0 \\
0 & -1 & 0 & 0 \\
-1 & 0 & 0 & 0
\end{array}\right], E^{2}=\left[\begin{array}{cccc}
0 & 0 & 1 & 0 \\
0 & 0 & 0 & -1 \\
-1 & 0 & 0 & 0 \\
0 & 1 & 0 & 0
\end{array}\right], } \\
E^{3} & =\left[\begin{array}{cccc}
0 & 1 & 0 & 0 \\
-1 & 0 & 0 & 0 \\
0 & 0 & 0 & 1 \\
0 & 0 & -1 & 0
\end{array}\right],
\end{aligned}
$$

satisfies

$E^{i} E^{j}=-\delta^{i j} I+\varepsilon^{i j k} E^{k}, \quad \varepsilon^{123}=1$. 
The zero modes of the T-dual metric and T-dual KalbRamond field (17) for the initial fields (138) are

${ }^{\star} G^{\mu \nu}=\left(G_{E}^{-1}\right)^{\mu \nu}=\frac{g}{g^{2}+b^{2}} I$

and

${ }^{\star} B^{\mu \nu}=\frac{\kappa}{2} \theta^{\mu \nu}=-\frac{1}{2} \frac{b^{i}}{g^{2}+b^{2}} E^{i}$,

with $b^{2}=b^{i} b^{i}$. They have the same form as the initial fields (138)

${ }^{\star} G_{\mu \nu}={ }^{\star} g \delta_{\mu \nu}, \quad{ }^{\star} B_{\mu \nu}={ }^{\star} b^{i} E_{\mu \nu}^{i}$,

with

${ }^{\star} g=\frac{g}{g^{2}+b^{2}}, \quad{ }^{\star} b=-\frac{b^{i}}{g^{2}+b^{2}}$.

One easily shows

${ }^{\star} g^{2}+{ }^{\star} b^{2}=\frac{1}{g^{2}+b^{2}}$.

In spheric coordinates one has

$$
\begin{aligned}
\left(g, b^{1}, b^{2}, b^{3}\right)= & (r \cos \theta, r \sin \theta \cos \varphi, \\
& \left.r \sin \theta \sin \varphi \cos \varphi_{1}, r \sin \theta \sin \varphi \sin \varphi_{1}\right),
\end{aligned}
$$

so $g^{2}+b^{2}=r^{2}$, and using (144) one obtains

$$
\begin{aligned}
\left({ }^{\star} g\right. & \left.{ }^{\star} b^{1},{ }^{\star} b^{2},{ }^{\star} b^{3}\right) \\
= & \left(\frac{1}{r} \cos \theta,-\frac{1}{r} \sin \theta \cos \varphi,-\frac{1}{r} \sin \theta \sin \varphi \cos \varphi_{1},\right. \\
& \left.-\frac{1}{r} \sin \theta \sin \varphi \sin \varphi_{1}\right) .
\end{aligned}
$$

Therefore, T-duality transforms $\left(r, \theta, \varphi, \varphi_{1}\right)$ to

$$
\left({ }^{\star} r,{ }^{\star} \theta,{ }^{\star} \varphi,{ }^{\star} \varphi_{1}\right)=\left(\frac{1}{r},-\theta, \varphi, \varphi_{1}\right) .
$$

From the relation $\Pi_{ \pm} G^{-1} \Pi_{\mp}=-\frac{1}{4} G_{E}$ we find

$$
\operatorname{det}\left(2 \Pi_{ \pm \mu \nu}\right)=\frac{g^{2}}{\star g^{2}}=\left(g^{2}+b^{2}\right)^{2}=r^{4} .
$$

Backgrounds corresponding to $r=1$ are mapped into themselves. The subset of this is the fixed surface with the condition

$\operatorname{det}\left(2 \Pi_{ \pm \mu \nu}\right)=r^{4}=1, \theta=0$

or $g=1, b^{i}=0$.

\section{Conclusion}

In this paper, we considered the closed string propagating in the weakly curved background (6), composed of a constant metric $G_{\mu \nu}$ and a linearly coordinate dependent Kalb-Ramond field $B_{\mu \nu}$, with infinitesimal field strength. We investigated the application of the generalized T-dualization procedure on the arbitrary set of coordinates and obtained the following T-duality diagram:

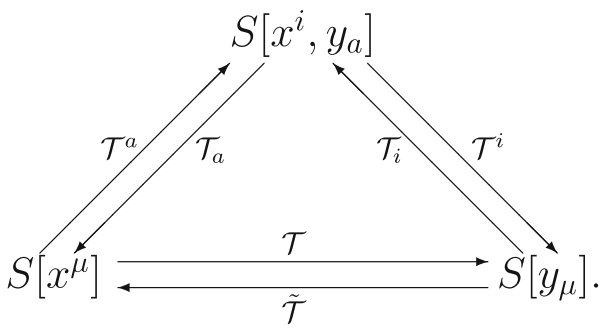

Let us stress that generalized T-dualization procedure enables the T-dualization along arbitrary direction, even if the background fields depend on these directions. The consequence of this procedure is that the arguments of the background fields, such as $\Delta V^{a}$, are non-local. They are non-local by definition, as they are the line integrals of the gauge fields. Once the explicit form is obtained the non-locality is seen in a fact that they depend on double coordinates $\tilde{x}$ and $\tilde{y}$, which are the line integrals of the $\tau$ and $\sigma$ derivatives of the original coordinates. To all the theories considered, except the initial theory, there corresponds the non-geometric, non-local flux.

The generalized T-dualization procedure was first applied along arbitrary $d(d=1, \ldots, D-1)$ coordinates $x^{a}=$ $\left\{x^{\mu_{1}}, \ldots, x^{\mu_{d}}\right\}$. We obtained the T-dual action $S\left[x^{i}, y_{a}\right]$, given by Eq. (36) with the dual background fields equal to

$$
\begin{aligned}
& \bullet \Pi_{+i j}=\bar{\Pi}_{+i j}, \quad \bullet \Pi_{+i}{ }^{a}=-\kappa \Pi_{+i b} \tilde{\Theta}_{-}^{b a}, \\
& \bullet \Pi_{+i}^{a}=\kappa \tilde{\Theta}_{-}^{a b} \Pi_{+b i}, \quad \cdot \Pi_{+}^{a b}=\frac{\kappa}{2} \tilde{\Theta}_{-}^{a b} .
\end{aligned}
$$

The argument of all background fields, $\left[x^{i}, V^{a}\left(x^{i}, y_{a}\right)\right]$, depends nonlinearly on coordinates $x^{i}, y_{a}$ through their doubles $\tilde{x}^{i}, \tilde{y}_{a}$ [see (39) and (40)]. All actions $S\left[x^{i}, y_{a}\right]$ are physically equivalent, but they are described with coordinates $x^{i}=\left\{x^{\mu_{d+1}}, \ldots, x^{\mu_{D}}\right\}$, for the untreated directions and dual coordinates $y_{a}=\left\{y_{\mu_{1}}, \ldots, y_{\mu_{d}}\right\}$, for the dualized directions. The case $d=D$ corresponds to the completely T-dual action with the T-dual fields $\frac{\kappa}{2} \Theta_{-}^{\mu \nu}(V(y))$ and the case $d=0$ to the initial action with the background $\Pi_{+\mu \nu}(x)$.

Applying the procedure to the T-dual action along dual directions $y_{a}=\left\{y_{\mu_{1}}, \ldots, y_{\mu_{d}}\right\}$ we obtained the initial theory, and applying it to the untreated directions $x^{i}=$ $\left\{x^{\mu_{d+1}}, \ldots, x^{\mu_{D}}\right\}$ we obtained the completely T-dual theory. All these derivations confirmed that the set of all Tdualizations forms an Abelian group. The neutral element 
of the group is the unexecuted T-dualization, while the Tdualizations along some subset of original directions $\mathcal{T}^{a}$ is inverse to the T-dualizations along the set of the corresponding dual directions $\mathcal{T}_{a}$.

Open Access This article is distributed under the terms of the Creative Commons Attribution 4.0 International License (http://creativecomm ons.org/licenses/by/4.0/), which permits unrestricted use, distribution, and reproduction in any medium, provided you give appropriate credit to the original author(s) and the source, provide a link to the Creative Commons license, and indicate if changes were made. Funded by SCOAP ${ }^{3}$.

\section{Appendix A: The background field compositions}

The background field compositions $\Pi_{ \pm \mu \nu}$ of the initial theory are

$\Pi_{ \pm \mu \nu}=B_{\mu \nu} \pm \frac{1}{2} G_{\mu \nu}$

where $G_{\mu \nu}$ and $B_{\mu \nu}$ are the initial metric and the initial KalbRamond field. The background field compositions $\Theta_{ \pm}^{\mu v}$ of the T-dual theory are

$\Theta_{ \pm}^{\mu \nu} \equiv-\frac{2}{\kappa}\left(G_{E}^{-1} \Pi_{ \pm} G^{-1}\right)^{\mu \nu}=\theta^{\mu \nu} \mp \frac{1}{\kappa}\left(G_{E}^{-1}\right)^{\mu \nu}$,

with $G_{E \mu \nu}$ being the effective metric

$G_{E \mu \nu} \equiv G_{\mu \nu}-4\left(B G^{-1} B\right)_{\mu \nu}$

and $\theta^{\mu v}$ being the parameter of non-commutativity

$\theta^{\mu \nu} \equiv-\frac{2}{\kappa}\left(G_{E}^{-1} B G^{-1}\right)^{\mu \nu}$.

These background field compositions satisfy

$\Pi_{ \pm \mu \nu} \Theta_{\mp}^{\nu \rho}=\Theta_{ \pm}^{\rho v} \Pi_{\mp v \mu}=\frac{1}{2 \kappa} \delta_{\mu}^{\rho}$.

Let us define the analogs of $\Theta_{ \pm}^{\mu v}$ in the $d$ - and $D-d$ dimensional subspaces determined by coordinates $x^{a}=$ $\left\{x^{\mu_{1}}, \ldots, x^{\mu_{d}}\right\}$ and $x^{i}=\left\{x^{\mu_{d+1}}, \ldots, x^{\mu_{D}}\right\}$, where $d=$ $1,2, \ldots, D-1$. The effective metrics in these subspaces are defined by

$$
\begin{aligned}
\tilde{G}_{E a b} & \equiv G_{a b}-4 B_{a c}\left(\tilde{G}^{-1}\right)^{c d} B_{d b}, \\
\bar{G}_{E i j} & \equiv G_{i j}-4 B_{i k}\left(\bar{G}^{-1}\right)^{k l} B_{l j},
\end{aligned}
$$

where $\tilde{G}_{a b} \equiv G_{a b}$ and $\bar{G}_{i j} \equiv G_{i j}$. Using these we define the following field compositions:

$$
\begin{aligned}
\tilde{\Theta}_{ \pm}^{a b} & \equiv-\frac{2}{\kappa}\left(\tilde{G}_{E}^{-1}\right)^{a c} \Pi_{ \pm c d}\left(\tilde{G}^{-1}\right)^{d b} \\
\bar{\Theta}_{ \pm}^{i j} & \equiv-\frac{2}{\kappa}\left(\bar{G}_{E}^{-1}\right)^{i k} \Pi_{ \pm k l}\left(\bar{G}^{-1}\right)^{l j}
\end{aligned}
$$

which are in fact the inverses of $2 \kappa \Pi_{\mp a b}$ and $2 \kappa \Pi_{\mp i j}$

$$
\begin{aligned}
\tilde{\Theta}_{ \pm}^{a b} \Pi_{\mp b c} & =\Pi_{\mp c b} \tilde{\Theta}_{ \pm}^{b a}=\frac{1}{2 \kappa} \delta_{c}^{a}, \\
\bar{\Theta}_{ \pm}^{i j} \Pi_{\mp j k} & =\Pi_{\mp k j} \bar{\Theta}_{ \pm}^{j i}=\frac{1}{2 \kappa} \delta_{k}^{i} .
\end{aligned}
$$

Analogously as the fields theta $\Theta_{ \pm}^{\mu \nu}$ defined in the whole space by (A.2), the theta fields defined in the subspaces can be separated into antisymmetric and symmetric parts as

$$
\begin{aligned}
\tilde{\Theta}_{ \pm}^{a b} & =\tilde{\theta}^{a b} \mp \frac{1}{\kappa}\left(\tilde{G}_{E}^{-1}\right)^{a b}, \\
\bar{\Theta}_{ \pm}^{i j} & =\bar{\theta}^{i j} \mp \frac{1}{\kappa}\left(\bar{G}_{E}^{-1}\right)^{i j},
\end{aligned}
$$

where

$$
\begin{aligned}
\tilde{\theta}^{a b} & \equiv-\frac{2}{\kappa}\left(\tilde{G}_{E}^{-1}\right)^{a c} B_{c d}\left(\tilde{G}^{-1}\right)^{d b}, \\
\bar{\theta}^{i j} & \equiv-\frac{2}{\kappa}\left(\bar{G}_{E}^{-1}\right)^{i k} B_{k l}\left(\bar{G}^{-1}\right)^{l j} .
\end{aligned}
$$

In the zeroth order the quantities $\Pi_{ \pm \mu \nu}, \Theta_{ \pm}^{\mu \nu}, \widetilde{\Theta}_{ \pm}^{a b}$, and $\bar{\Theta}_{ \pm}^{i j}$ reduce to

$$
\begin{aligned}
\Pi_{0 \pm \mu \nu} & =b_{\mu \nu} \pm \frac{1}{2} G_{\mu \nu}, \\
\Theta_{0 \pm}^{\mu \nu} & =-\frac{2}{\kappa}\left(g^{-1}\right)^{\mu \rho} \Pi_{0 \pm \rho \sigma}\left(G^{-1}\right)^{\sigma \nu}=\theta_{0}^{\mu \nu} \mp \frac{1}{\kappa}\left(g^{-1}\right)^{\mu \nu}, \\
\widetilde{\Theta}_{0 \pm}^{a b} & =-\frac{2}{\kappa}\left(\tilde{g}^{-1}\right)^{a c} \Pi_{0 \pm c d}\left(\tilde{G}^{-1}\right)^{d b}=\tilde{\theta}_{0}^{a b} \mp \frac{1}{\kappa}\left(\tilde{g}^{-1}\right)^{a b}, \\
\bar{\Theta}_{0 \pm}^{i j} & =-\frac{2}{\kappa}\left(\bar{g}^{-1}\right)^{i k} \Pi_{0 \pm k l}\left(\bar{G}^{-1}\right)^{l j}=\bar{\theta}_{0}^{i j} \mp \frac{1}{\kappa}\left(\bar{g}^{-1}\right)^{i j},
\end{aligned}
$$

where the zeroth order effective metrics are

$$
\begin{aligned}
g_{\mu \nu} & =G_{\mu \nu}-4 b_{\mu \rho}\left(G^{-1}\right)^{\rho \sigma} b_{\sigma \nu}, \\
\tilde{g}_{a b} & =G_{a b}-4 b_{a c}\left(\tilde{G}^{-1}\right)^{c d} b_{d b}, \\
\bar{g}_{i j} & =G_{i j}-4 b_{i k}\left(\bar{G}^{-1}\right)^{k l} b_{l j},
\end{aligned}
$$

and the zeroth order non-commutativity parameters are

$$
\begin{aligned}
\theta_{0}^{\mu \nu} & =-\frac{2}{\kappa}\left(g^{-1}\right)^{\mu \rho} b_{\rho \sigma}\left(G^{-1}\right)^{\sigma \nu}, \\
\tilde{\theta}_{0}^{a b} & =-\frac{2}{\kappa}\left(\tilde{g}^{-1}\right)^{a c} b_{c d}\left(\tilde{G}^{-1}\right)^{d b} \\
\bar{\theta}_{0}^{i j} & =-\frac{2}{\kappa}\left(\bar{g}^{-1}\right)^{i k} b_{k l}\left(\bar{G}^{-1}\right)^{l j}
\end{aligned}
$$


Quantities $\Pi_{0 \pm \mu \nu}, \Theta_{0 \pm}^{\mu \nu}, \widetilde{\Theta}_{0 \pm}^{a b}$, and $\bar{\Theta}_{0 \pm}^{i j}$ satisfy

$\Pi_{0 \pm \mu \nu} \Theta_{0 \mp}^{v \rho}=\Theta_{0 \pm}^{\rho v} \Pi_{0 \mp \nu \mu}=\frac{1}{2 \kappa} \delta_{\mu}^{\rho}$,

$\Pi_{0 \pm a b} \tilde{\Theta}_{0 \mp}^{b c}=\tilde{\Theta}_{0 \pm}^{c b} \Pi_{0 \mp b a}=\frac{1}{2 \kappa} \delta_{a}^{c}$,

$\Pi_{0 \pm i j} \bar{\Theta}_{0 \mp}^{j k}=\bar{\Theta}_{0 \pm}^{k j} \Pi_{0 \mp j i}=\frac{1}{2 \kappa} \delta_{i}^{k}$.

The non-commutativity parameters theta $\Theta_{ \pm}^{\mu \nu}, \tilde{\Theta}_{ \pm}^{a b}$, and $\bar{\Theta}_{ \pm}^{i j}$ can be expressed as

$\Theta_{ \pm}^{\mu \nu}=\Theta_{0 \pm}^{\mu v}-2 \kappa \Theta_{0 \pm}^{\mu \rho} h_{\rho \sigma} \Theta_{0 \pm}^{\sigma v}$,

$\tilde{\Theta}_{ \pm}^{a b}=\tilde{\Theta}_{0 \pm}^{a b}-2 \kappa \tilde{\Theta}_{0 \pm}^{a c} h_{c d} \tilde{\Theta}_{0 \pm}^{d b}$,

$\bar{\Theta}_{ \pm}^{i j}=\bar{\Theta}_{0 \pm}^{i j}-2 \kappa \bar{\Theta}_{0 \pm}^{i k} h_{k l} \bar{\Theta}_{0 \pm}^{l j}$.

Appendix A.1: Relations between field compositions

In Sect. 3.2 we introduced the background field composition

$\bar{\Pi}_{ \pm i j} \equiv \Pi_{ \pm i j}-2 \kappa \Pi_{ \pm i a} \tilde{\Theta}_{\mp}^{a b} \Pi_{ \pm b j}$

and analogously we define

$\widetilde{\Pi}_{ \pm a b} \equiv \Pi_{ \pm a b}-2 \kappa \Pi_{ \pm a i} \bar{\Theta}_{\mp}^{i j} \Pi_{ \pm j b}$.

Here we will show that these quantities are the inverses of the ordinary non-commutativity parameters theta, projected to the $i$ - and $a$-subspaces [see (A.22)].

Let us express the tensors $\Pi_{ \pm \mu \nu}$ and $\Theta_{ \pm}^{\mu \nu}$, which satisfy (A.5), in a block-wise form as

$\Pi_{ \pm \mu \nu}=\left(\begin{array}{cc}\Pi_{ \pm i j} & \Pi_{ \pm i b} \\ \Pi_{ \pm a j} & \Pi_{ \pm a b}\end{array}\right), \quad \Theta_{ \pm}^{\mu v}=\left(\begin{array}{cc}\Theta_{ \pm}^{i j} & \Theta_{ \pm}^{i b} \\ \Theta_{ \pm}^{a j} & \Theta_{ \pm}^{a b}\end{array}\right)$

We will use the definition of block-wise inversion, which states that the inverse of the matrix of the form

$M=\left(\begin{array}{ll}A & B \\ C & D\end{array}\right)$

equals

$$
\begin{aligned}
& M^{-1} \\
& \quad=\left(\begin{array}{cc}
\left(A-B D^{-1} C\right)^{-1} & -A^{-1} B\left(D-C A^{-1} B\right)^{-1} \\
-D^{-1} C\left(A-B D^{-1} C\right)^{-1} & \left(D-C A^{-1} B\right)^{-1}
\end{array}\right) .
\end{aligned}
$$

Applying (A.20) to the first matrix in (A.18), Eq. (A.5) implies

$2 \kappa \Theta_{\mp}^{i j}=\left(\Pi_{ \pm i j}-2 \kappa \Pi_{ \pm i a} \tilde{\Theta}_{\mp}^{a b} \Pi_{ \pm b j}\right)^{-1}$,

$2 \kappa \Theta_{\mp}^{i b}=-2 \kappa \bar{\Theta}_{\mp}^{i j} \Pi_{ \pm j a}\left(\Pi_{ \pm a b}-2 \kappa \Pi_{ \pm a k} \bar{\Theta}_{\mp}^{k l} \Pi_{ \pm l b}\right)^{-1}$,
$2 \kappa \Theta_{\mp}^{a j}=-2 \kappa \tilde{\Theta}_{\mp}^{a b} \Pi_{ \pm b i}\left(\Pi_{ \pm i j}-2 \kappa \Pi_{ \pm i c} \tilde{\Theta}_{\mp}^{c d} \Pi_{ \pm d j}\right)^{-1}$,

$2 \kappa \Theta_{\mp}^{a b}=\left(\Pi_{ \pm a b}-2 \kappa \Pi_{ \pm a i} \bar{\Theta}_{\mp}^{i j} \Pi_{ \pm j b}\right)^{-1}$,

and we can conclude that (A.16) and (A.17) are the inverses of $2 \kappa \Theta_{\mp}^{i j}$ and $2 \kappa \Theta_{\mp}^{a b}$, respectively. So, we can write

$\bar{\Pi}_{ \pm i j} \Theta_{\mp}^{j k}=\Theta_{\mp}^{k j} \bar{\Pi}_{ \pm j i}=\frac{1}{2 \kappa} \delta_{i}^{k}$,

$\widetilde{\Pi}_{ \pm a b} \Theta_{\mp}^{b c}=\Theta_{\mp}^{c b} \widetilde{\Pi}_{ \pm b a}=\frac{1}{2 \kappa} \delta_{a}^{c}$,

and

$\Theta_{\mp}^{i b}=-2 \kappa \Theta_{\mp}^{i j} \Pi_{ \pm j a} \Theta_{\mp}^{a b}$,

$\Theta_{\mp}^{a j}=-2 \kappa \tilde{\Theta}_{\mp}^{a b} \Pi_{ \pm b i} \Theta_{\mp}^{i j}$.

Applying (A.20) to the second matrix in (A.18), Eq. (A.5) implies

$$
\begin{aligned}
& 2 \kappa \Pi_{\mp i j}=\left(\Theta_{ \pm}^{i j}-2 \kappa \Theta_{ \pm}^{i a} \widetilde{\Pi}_{a b \mp} \Theta_{ \pm}^{b j}\right)^{-1} \\
& 2 \kappa \Pi_{\mp i b}=-2 \kappa \bar{\Pi}_{\mp i j} \Theta_{ \pm}^{j a}\left(\Theta_{ \pm}^{a b}-2 \kappa \Theta_{ \pm}^{a k} \bar{\Pi}_{\mp k l} \Theta_{ \pm}^{l b}\right)^{-1} \\
& 2 \kappa \Pi_{\mp a j}=-2 \kappa \widetilde{\Pi}_{a b \mp} \Theta_{ \pm}^{b i}\left(\Theta_{ \pm}^{i j}-2 \kappa \Theta_{ \pm}^{i c} \widetilde{\Pi}_{\mp c d} \Theta_{ \pm}^{d j}\right)^{-1} \\
& 2 \kappa \Pi_{\mp a b}=\left(\Theta_{ \pm}^{a b}-2 \kappa \Theta_{ \pm}^{a i} \bar{\Pi}_{\mp i j} \Theta_{ \pm}^{j b}\right)^{-1}
\end{aligned}
$$

so using (A.8) we conclude that

$\bar{\Theta}_{ \pm}^{i j}=\Theta_{ \pm}^{i j}-2 \kappa \Theta_{ \pm}^{i a} \widetilde{\Pi}_{a b \mp} \Theta_{ \pm}^{b j}$,

$\tilde{\Theta}_{ \pm}^{a b}=\Theta_{ \pm}^{a b}-2 \kappa \Theta_{ \pm}^{a i} \bar{\Pi}_{\mp i j} \Theta_{ \pm}^{j b}$,

and

$\Pi_{\mp i b}=-2 \kappa \bar{\Pi}_{\mp i j} \Theta_{ \pm}^{j a} \Pi_{\mp a b}$
$\Pi_{\mp a j}=-2 \kappa \widetilde{\Pi}_{\mp a b} \Theta_{ \pm}^{b i} \Pi_{\mp i j}$.

Let us derive some useful relations between these quantities. Equation (A.5), for $\mu=a, v=i$ and $\mu=i, v=a$, becomes

$\Pi_{ \pm a b} \Theta_{\mp}^{b i}=-\Pi_{ \pm a j} \Theta_{\mp}^{j i}$,

$\Pi_{ \pm i j} \Theta_{\mp}^{j a}=-\Pi_{ \pm i b} \Theta_{\mp}^{b a}$,

while taking $\mu=a, v=b$ and $\mu=i, v=j$ we obtain

$\Pi_{ \pm a c} \Theta_{\mp}^{c b}+\Pi_{ \pm a i} \Theta_{\mp}^{i b}=\frac{1}{2 \kappa} \delta_{a}^{b}$,

$\Pi_{ \pm i a} \Theta_{\mp}^{a j}+\Pi_{ \pm i k} \Theta_{\mp}^{k j}=\frac{1}{2 \kappa} \delta_{i}^{j}$.

Multiplying Eq. (A.27) from the left with $\tilde{\Theta}_{\mp}^{c a}$ and from the right with $\bar{\Pi}_{\mp i k}$ we get the relation

$\Theta_{\mp}^{c i} \bar{\Pi}_{ \pm i k}=-\tilde{\Theta}_{\mp}^{c a} \Pi_{ \pm a k}$,

while multiplying Eq. (A.28) from the right with $\bar{\Theta}_{\mp}^{k i}$ and from the left with $\tilde{\Pi}_{ \pm a c}$, we obtain

$\Theta_{\mp}^{k a} \widetilde{\Pi}_{ \pm a c}=-\bar{\Theta}_{\mp}^{k i} \Pi_{ \pm i c}$. 


\section{References}

1. A. Giveon, M. Porrati, E. Rabinovici, Phys. Rep. 244, 77-202 (1994)

2. E. Alvarez, L. Alvarez-Gaume, Y. Lozano, Nucl. Phys. Proc. Suppl. 41, 1 (1995)

3. B. Zwiebach, A First Course in String Theory, 2nd edn. (Cambridge University Press, 2009)

4. K. Becker, M. Becker, J.H. Schwarz, String Theory and MTheory-A Modern Introdution (Cambridge University Press, 2007)

5. T.H. Buscher, Phys. Lett. B 201(4), 466 (1988)

6. M. Roček, E. Verlinde, Nucl. Phys. B 373, 630 (1992)

7. J. Shelton, W. Taylor, B. Wecht, JHEP 10, 085 (2005)

8. R. Blumenhagen, A. Deser, D. Lüst, E. Plauschinn, F. Rennecke, J. Phys. A 44, 385401 (2011)

9. B. Nikolić, B. Sazdović, Phys. Rev. D 84, 065012 (2011)

10. B. Nikolić, B. Sazdović, JHEP 06, 101 (2012)

11. O. Hohm, Prog. Theor. Phys. Suppl. 188, 116-125 (2011)
12. C. Hull, B. Zwiebach, JHEP 09, 099 (2009)

13. O. Hohm, C. Hull, B. Zwiebach, JHEP 08, 008 (2010)

14. O. Hohm, C. Hull, B. Zwiebach, JHEP 07, 016 (2010)

15. D. Lüst, JHEP 12, 084 (2010)

16. D. Andriot, M. Larfors, D. Lüst, P. Patalong, JHEP 06, 021 (2013)

17. D. Andriot, O. Hohm, M. Larfors, D. Lüst, P. Patalong, Phys. Rev. Lett. 108, 261602 (2012)

18. Lj. Davidović, B. Sazdović, EPJC 74(1), 2683 (2014)

19. Lj. Davidović, B. Nikolić, B. Sazdović, EPJC 74(1), 2734 (2014)

20. A. Giveon, E. Rabinovici, G. Veneciano, Nucl. Phys. B 323, 167 (1989)

21. Lj. Davidović, B. Sazdović, Phys. Rev. D 83, 066014 (2011)

22. Lj. Davidović, B. Sazdović, JHEP 08, 112 (2011)

23. Lj. Davidović, B. Sazdović, EPJC 72(11), 2199 (2012)

24. N. Seiberg, E. Witten, JHEP 09, 032 (1999)

25. B. Nikolić, B. Sazdović, Nucl. Phys. B 836, 100 (2010)

26. B. Sazdović, JHEP 08, 055 (2015)

27. R. Blumenhagen, E. Plauschinn, J. Phys. A Math. Theor. 44, 015401 (2011) 